\title{
Biovolume de cianobactérias e algas de reservatórios tropicais do Brasil com diferentes estados tróficos
}

\author{
Bárbara Medeiros Fonseca ${ }^{1,6}$, Carla Ferragut ${ }^{2}$, Andréa Tucci ${ }^{3}$, Luciane Oliveira Crossetti ${ }^{4}$, Fernanda Ferrari ${ }^{5}$, \\ Denise de Campos Bicudo², Célia Leite Sant'Anna ${ }^{3}$ e Carlos Eduardo de Mattos Bicudo ${ }^{2}$
}

Recebido: 5.12.2012; aceito: 16.08 .2013

\begin{abstract}
Biovolume of Cyanobacteria and algae from Brazilian tropical reservoirs with different trophic status). The present study aimed at presenting the biovolume of planktonic and periphytic cyanobacteria and algae from reservoirs with different trophic status at Parque Estadual das Fontes do Ipiranga, São Paulo, São Paulo State, Brazil. The study includes a proposal for determining the biovolume of colonial forms of Cyanobacteria such as Microcystis and Sphaerocavum. The list of biovolume will contribute to algal ecology studies, allowing the application of this information to research in this area of knowledge, besides serving as a comparative model for other tropical ecosystems. The list comprises the biovolume of 568 taxa, including information on their taxonomic classes, maximum linear dimension and surface/volume ratio.
\end{abstract}

Keywords: phycoperiphyton, phytoplankton, quantitative studies

RESUMO - (Biovolume de cianobactérias e algas de reservatórios tropicais do Brasil com diferentes estados tróficos). O presente estudo visou apresentar o biovolume de cianobactérias e algas fitoplanctônicas e perifíticas de reservatórios com diferentes estados tróficos do Parque Estadual das Fontes do Ipiranga, São Paulo, SP, Brasil. O estudo inclui uma proposta para determinação do biovolume de formas coloniais de Cyanobacteria como, por exemplo, Microcystis e Sphaerocavum. A lista de biovolume auxiliará os estudos sobre ecologia de algas, permitindo a aplicação dessa informação em trabalhos nessa área de estudo, além de servir de modelo comparativo em outros ecossistemas de região tropical. A lista inclui o biovolume de 568 táxons, incluindo informações sobre suas respectivas classes taxonômicas, máxima dimensão linear e razão superfície/volume.

Palavras-chave: estudos quantitativos, ficoperifíton, fitoplâncton

\section{Introdução}

O biovolume é um atributo estrutural básico das comunidades algais, que pode ser potencialmente usado para acessar condições ambientais (Stevenson \& Smol 2003). O tamanho das algas varia consideravelmente de uma espécie para outra e em uma mesma espécie e, ainda, ao longo do crescimento durante diferentes fases do ciclo de vida (Bellinger \& Sigee 2010). O significado ecológico do tamanho das algas em relação à sua biomassa pode ser observado quando se compara a participação de espécies de dimensões máximas lineares muito distintas na estrutura de uma comunidade (Bellinger \& Sigee 2010). Por exemplo, a contribuição de um único indivíduo de uma espécie microplanctônica pode ser equivalente à de vários indivíduos de uma espécie picoplanctônica. Do mesmo modo, espécies coloniais ou filamentosas consideradas como unidades na contagem acabam tendo sua abundância relativa subestimada (Figueredo \& Giani 2001). Tal fato pode apresentar uma série de implicações ecológicas, pois tais diferenças de tamanho estão diretamente relacionadas à forma como as populações exploram os recursos ambientais disponíveis (Reynolds 1984). Por esses

1. Universidade Católica de Brasília, Curso de Ciências Biológicas (Sala E-10), QS 07, Lote 1, Águas Claras, $71966-700$ Brasília, DF, Brasil

2. Instituto de Botânica, Núcleo de Pesquisa em Ecologia, Caixa Postal 68041, 04045-972 São Paulo, SP, Brasil

3. Instituto de Botânica, Núcleo de Pesquisa em Ficologia, Caixa Postal 68041, 04045-972 São Paulo, SP, Brasil

4. Universidade Federal do Rio Grande do Sul, Departamento de Ecologia, Caixa Postal 15007, 91501-970 Porto Alegre, RS, Brasil

5. Universidade Tecnológica Federal do Paraná, Câmpus Dois Vizinhos, Estrada para Boa Esperança, Km 04, 85660-000 Dois Vizinhos, PR, Brasil

6. Autor para correspondência: barbara.fonseca0603@gmail.com 
motivos, o biovolume tem sido o atributo estrutural mais utilizado para expressar quantitativamente as comunidades fitoplanctônicas e ficoperifíticas em estudos ecológicos (ex. Soares et al. 2007, Crossetti \& Bicudo 2008, Kruk et al. 2009, Becker et al. 2010, Wyatt et al. 2010, Fonseca \& Bicudo 2011, Borduqui \& Ferragut 2012).

Conforme Bellinger \& Sigee (2010), o biovolume do fitoplâncton pode ser considerado em relação aos seguintes aspectos: (1) biovolume médio da espécie, que consiste no volume médio de organismos individuais (células ou colônias), expresso em micrômetros cúbicos $\left(\mu \mathrm{m}^{3}\right)$; (2) biovolume populacional, ou seja, o volume ocupado por uma única população por unidade de volume da água do lago, expresso em micrômetros cúbicos por litro $\left(\mu \mathrm{m}^{3} \mathrm{~L}^{-1}\right)$; e (3) biovolume total do fitoplâncton, ou o volume acumulado ocupado por todas as espécies de fitoplâncton em uma amostra, expresso em micrômetros cúbicos por litro $\left(\mu \mathrm{m}^{3} \mathrm{~L}^{-1}\right)$. Para a comunidade ficoperifítica, o biovolume é comumente expresso por unidade de área de substrato (ex. micrômetros cúbicos por centímetro quadrado $\mu \mathrm{m}^{3} \mathrm{~cm}^{-2}$ ) (Stevenson 1996).

O cálculo do biovolume é normalmente feito tomando-se como base os sólidos geométricos que mais se aproximem, isolados ou combinados, da forma da célula. Trabalhos como os de Edler (1979), Rott (1981), Hillebrand et al. (1999) e Sun \& Liu (2003) apresentaram fórmulas a serem utilizadas para vários táxons. Alguns trabalhos trazem, às vezes, propostas de novos modelos geométricos para o cálculo do biovolume de espécies em particular (ex. Konoplya \& Soares 2011), evidenciando a necessidade de um constante aprimoramento dos cálculos. Outros ainda sugerem a necessidade de padronização dos cálculos devido à influência de fixadores na redução do biovolume algal (Hawkins et al. 2005).

Outra possibilidade para determinação do biovolume algal é a utilização de programas de computador desenvolvidos especificamente para os estudos quantitativos, os quais trazem opções de cálculo de biovolume. Nesses casos, é necessária apenas a seleção da forma mais apropriada para a espécie e a inserção de suas medidas. Alguns exemplos destes programas são o Algamica (Gosselain \& Hamilton 2000) e o OptiCount (Hepperle \& Schmidt-Halewicz 2000). O Algamica foi criado para quantificação de algas planctônicas, perifíticas e bentônicas e sua última versão dispõe de 20 formas geométricas para cálculo de biovolume. O OptiCount foi desenvolvido para enumeração de plâncton e partículas em geral e dispõe de 23 formas geométricas, sendo uma delas livre para a inserção de um valor de biovolume fixo, previamente calculado. Contudo, nem sempre as formas geométricas mais apropriadas para algumas espécies são fornecidas nos programas de quantificação, o que leva a estimativas muitas vezes grosseiras de biovolume.

Dados de biovolume para fins comparativos em ecossistemas subtropicais brasileiros são escassos (ex. Torgan et al. 1998). Por sua vez, aqueles referentes a ambientes tropicais estão distribuídos, de modo geral, em trabalhos de dissertações e teses, jamais publicados. Frequentemente os dados brutos dos biovolumes de cada espécie ficam restritos aos apêndices de tais trabalhos acadêmicos. Nesse contexto, este estudo teve como objetivo apresentar o biovolume de algas planctônicas e perifíticas de reservatórios com diferentes estados tróficos localizados no Parque Estadual das Fontes do Ipiranga, São Paulo, SP, Brasil.

\section{Material e métodos}

Área de estudo - Os reservatórios estudados são três sistemas aquáticos localmente chamados Lago das

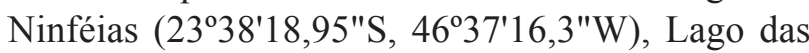

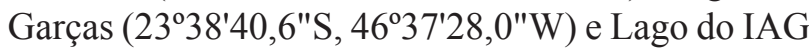

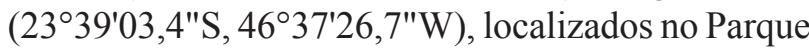
Estadual das Fontes do Ipiranga (PEFI), região sul do Município de São Paulo, em área sob jurisdição do Instituto de Botânica da Secretaria do Meio Ambiente do Estado de São Paulo. O Lago das Ninfeias $\left(\mathrm{Z}_{\text {máx }}=3,6 \mathrm{~m} ; \mathrm{Z}_{\text {med }}=1,32 \mathrm{~m}\right.$; área $\left.=5.433 \mathrm{~m}^{2}\right)$ é um reservatório com características oligo-mesotróficas, que apresenta região litorânea com grande abundância de macrófitas aquáticas (Fonseca \& Bicudo 2010, 2011). O Lago das Garças $\left(Z_{\text {máx }}=4,7 \mathrm{~m} ; Z_{\text {med }}=2,14 \mathrm{~m}\right.$; área $=88.156 \mathrm{~m}^{2}$ ) é um reservatório com características eutróficas, elevado aporte de matéria orgânica e floração periódica de cianobactérias (Crossetti \& Bicudo 2008, Fonseca \& Bicudo 2008). O Lago do IAG (Instituto de Ciências Atmosféricas e Geofísicas) $\left(\mathrm{Z}_{\text {máx }}=4,7 \mathrm{~m} ; \mathrm{Z}_{\text {med }}=1,51 \mathrm{~m}\right.$; área $\left.=11.270 \mathrm{~m}^{2}\right)$ é, por sua vez, o único oligotrófico dentro da unidade de conservação (Lopes et al. 2005).

Amostragem - Amostras da comunidade de algas planctônicas e perifíticas foram coletadas nos reservatórios do PEFI para os estudos de Tucci (2002), Ferragut (2004), Fonseca (2005), Crossetti (2006) e Ferrari (2010). 
Inicialmente, calculou-se o biovolume individual de cada táxon a partir dos valores médios das medidas de um até 83 indivíduos, dependendo do tamanho da população analisada. O material analisado encontrava-se já fixado em solução de formalina a 4\% ou em lugol acético. Para o presente estudo, fez-se uma média dos dados encontrados nos trabalhos citados acima, para cada táxon. Os cálculos de biovolume basearam-se nas fórmulas sugeridas por Hillebrand et al. (1999) e Sun \& Liu (2003), às vezes com algumas adaptações.

A tabela 1 apresenta as formas utilizadas, com suas respectivas legendas. No caso de espécies de cianobactérias com colônias densas, tais como as de Microcystis (exceto M. protocystis) e Sphaerocavum, os biovolumes foram calculados em três ou quatro categorias diferentes, considerando colônias pequenas $(\mathrm{P})$, médias $(\mathrm{M})$, grandes $(\mathrm{G})$ ou muito grandes $(\mathrm{GG})$ (tabela 2).

Para estas cianobactérias, o biovolume foi calculado para cada categoria segundo os passos a seguir:

1) Calculou-se o biovolume total da colônia, excetuada a mucilagem, como se a colônia fosse um sólido compacto. Em geral, foram usadas formas geométricas como esfera, esferoide, elipsoide ou a combinação dos mesmos (figura 1).

2) Multiplicou-se o biovolume total da colônia por um fator (f) definido arbitrariamente, o qual dependeu da espécie considerada. Tal fator considerou o espaço livre entre as células, que é uma característica taxonômica de cada espécie. Exemplo:

Microcystis aeruginosa: $\mathrm{f}=0,7 \rightarrow \mathrm{V}_{\text {final }}=\mathrm{V}_{\text {total }} \times 0,7$ Microcystis panniformis: $\mathrm{f}=0,9 \rightarrow \mathrm{V}_{\text {final }}=\mathrm{V}_{\text {total }} \times 0,9$

3) Calculou-se o biovolume celular individual (esfera): $\mathrm{V}_{\text {cél }}=(\pi / 6) \times \mathrm{a}^{3}$

4) O número de células da colônia foi estimado dividindo-se o biovolume da colônia (passo 2 pelo biovolume da célula (passo 3).

5) Para espécies como Sphaerocavum brasiliense, que apresenta colônias ocas, o biovolume relacionado ao espaço central vazio deve ser subtraído do valor total da colônia durante o passo 1 (figura 2). Os espaços entre as células foram compensados multiplicando-se o biovolume total pelo fator 0,7 (passo 2):

S. brasiliense: $\mathrm{f}=0,7 \rightarrow \mathrm{V}_{\text {final }}=\mathrm{V}_{\text {total }} \times 0,7$

\section{Resultados e Discussão}

São apresentados, ao todo, os biovolumes de 568 táxons (espécies, variedades e formas taxonômicas) de cianobactérias e algas, além de suas respectivas classes taxonômicas, máxima dimensão linear e razão superfície/volume (tabela 3). Destaca-se o número relativamente alto de táxons mencionados no presente estudo. A título de comparação, o trabalho de Torgan et al. (1998), um dos poucos publicados no Brasil sobre o biovolume de espécies fitoplanctônicas, apresentou os dados de 37 táxons.

O presente estudo propõe uma nova forma de cálculo do biovolume de cianobactérias coloniais. Várias destas espécies frequentemente formam

Tabela 1. Formas geométricas utilizadas no estudo de táxons fitoplanctônicos e ficoperifíticos do Parque Estadual das Fontes do Ipiranga, São Paulo, SP, Brasil, e suas respectivas legendas. As fórmulas podem ser conferidas em Hillebrand et al. (1999) e Sun \& Liu (2003).

Table 1. Geometric forms used in the study of phytoplanktonic and phycoperiphytic taxa from Parque Estadual das Fontes do Ipiranga, São Paulo, São Paulo State, Brazil, and their respective legends. Formulas can be checked in Hillebrand et al. (1999) and Sun \& Liu (2003).

\begin{tabular}{ll}
\hline Forma geométrica & Legenda \\
\hline 2 cilindros +2 cones & 2 cil +2 cone \\
2 cones & 2 cone \\
2 cones truncados & 2 cone tr \\
2 cones truncados +2 cilindros & 2 cone $\mathrm{tr}+2$ cil \\
2 cones truncados +4 cilindros & 2 cone tr +4 cil \\
2 cones truncados +6 cilindros & 2 cone tr +6 cil \\
2 primas retangulares & 2 Pret \\
Cilindro & Cil \\
Cilindro +2 cones & cil +2 cone \\
Cilindro +2 cones $(\mathrm{H}=1,2 \mathrm{~h})$ & cil +2 cone* \\
Cimbelóide & Cimb \\
Cone & Cone \\
Cone + semi-esfera & cone $+0,5$ esf \\
Elipsoide & Elip \\
Esfera & Esf \\
Esfera externa - esfera interna $(\mathrm{x} 0,7)$ & esfext - esfint* \\
Esferoide & Esfo \\
Gomphonemoide & Gomph \\
Monoraphidioide & Mon \\
Prisma semi-elíptico & P0,5elip \\
Prisma elíptico & Peli \\
Prisma foice & Pfoi \\
Pirâmide & Pir \\
Prisma losangular & Plos \\
Prisma retangular & Pret \\
Prisma retangular +8 cones & Pret +8 cone \\
\hline
\end{tabular}


Tabela 2. Máximas dimensões lineares $(\mu \mathrm{m})$ utilizadas no estudo de táxons fitoplanctônicos e ficoperifíticos do Parque Estadual das Fontes do Ipiranga, São Paulo, SP, Brasil, para definição de colônias pequenas $(\mathrm{P})$, médias $(\mathrm{M})$, grandes (G) e muito grandes (GG) nos indivíduos de Microcystis e Sphaerocavum.

Table 2. Greatest axis linear dimension $(\mu \mathrm{m})$ used in the study of phytoplanktonic and phycoperiphytic taxa from Parque Estadual das Fontes do Ipiranga, São Paulo, São Paulo State, Brazil, in the definition of small (S), medium (M), large (L), and very large (VL) colonies of Microcystis and Sphaerocavum.

\begin{tabular}{|c|c|c|c|c|}
\hline & & $\begin{array}{l}\text { Microcystis } \\
\text { aeruginosa }\end{array}$ & $\begin{array}{l}\text { Microcystis } \\
\text { panniformis }\end{array}$ & $\begin{array}{l}\text { phaerocavum } \\
\text { brasiliense }\end{array}$ \\
\hline \multirow{3}{*}{$\mathrm{P}$} & média & 27,3 & 49,6 & 26,5 \\
\hline & mínimo & 19,0 & 34,2 & 13,2 \\
\hline & máximo & 50,0 & 65,0 & 47,9 \\
\hline \multirow{3}{*}{$\mathrm{M}$} & média & 42,1 & 77,0 & 69,6 \\
\hline & mínimo & 30,0 & - & 40,5 \\
\hline & máximo & 53,2 & - & 122,3 \\
\hline \multirow{3}{*}{ G } & média & 111,7 & 168,2 & 120,2 \\
\hline & mínimo & 75,0 & 100,0 & 64,3 \\
\hline & máximo & 200,0 & 235,8 & 198,2 \\
\hline \multirow{3}{*}{$\mathrm{GG}$} & média & - & - & 314,5 \\
\hline & mínimo & - & - & 183,7 \\
\hline & máximo & - & - & 420,0 \\
\hline
\end{tabular}

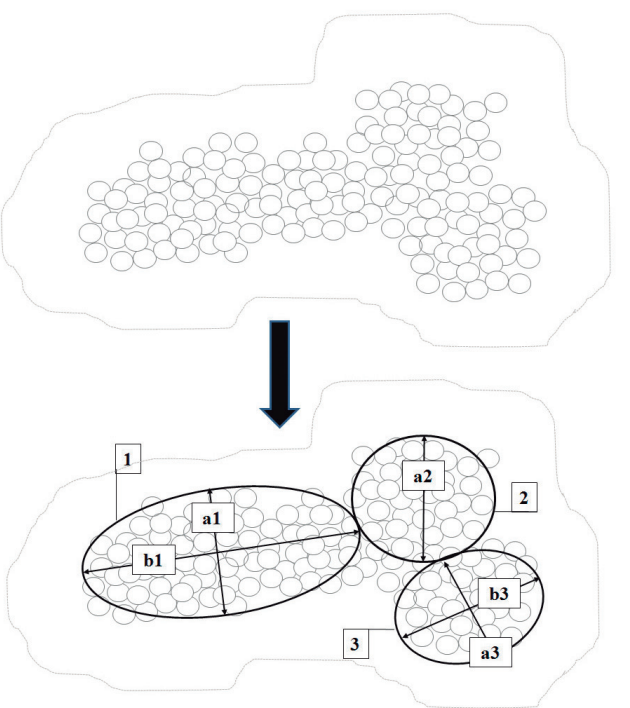

Figura 1. Esquema de uma colônia de Microcystis fragmentada em formas geométricas independentes (1: elipsoide, 2: esfera, 3: elipsoide) para o cálculo do biovolume.

Figure 1. Schema of a Microcystis colony fragmented in independent geometric forms (1: ellipsoid, 2: sphere, 3: ellipsoid) for the biovolume calculation. florações e são muito citadas por sua toxicidade (ex. Giani et al. 2005, Sant'Anna et al. 2008), razão pela qual a contagem de células de cianobactérias passou a ser um procedimento requerido pela legislação brasileira (Brasil 2011). As empresas de tratamento de água quantificam, em geral, as células de cianobactérias usando o retículo de Whipple, conforme proposto por Jardim et al. (2002), como segue: (1) contar o número médio de células em cada quadrado; (2) contar o número de quadrados sobrepostos pelas colônias; e (3) multiplicar o número de quadrados contados pelo número médio de células em cada quadrado. Em alguns casos, o número de células em um plano é multiplicado pelo fator 2 ou 3, considerando o plano de divisão da espécie (ex. fator 3 para espécies da família Microcystaceae e fator 2 para espécies da família Merismopediaceae). Entretanto, o presente estudo questiona tais procedimentos por considerar que subestimam o número de células numa dada colônia, em especial as maiores, formadoras de florações. Estas espécies apresentam colônias tridimensionais, o que torna muito difícil a quantificação precisa do número de células num único plano, especialmente em colônias mais densas como as de Microcystis panniformis.

Para Hillebrand et al. (1999), a estimativa do biovolume apresenta vários aspectos positivos, que o tornam uma medida recomendada de biomassa,

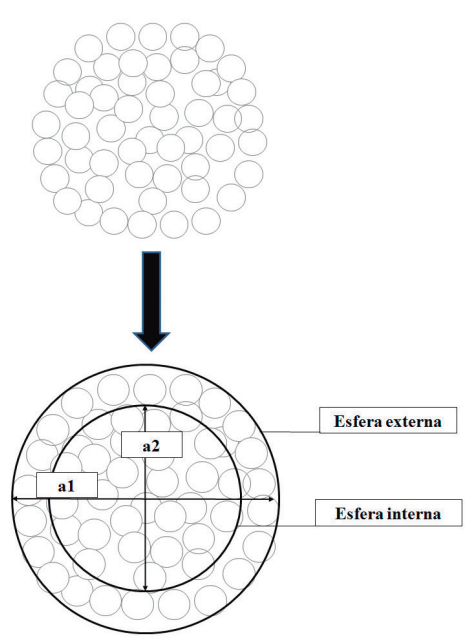

Figura 2. Esquema de uma colônia de Sphaerocavum detalhando o cálculo do biovolume a partir de uma esfera externa, com dimensão correspondente a "a1", da qual é subtraída a dimensão da esfera interna (correspondente a "a2").

Figure 2. Schema of a Sphaerocavum colony detailing the biovolume calculation of an external sphere, with dimension corresponding to "a1", from which the internal sphere dimensionis subtracted (corresponding to "a2"). 
Tabela 3. Biovolume $\left(\mathrm{BV}, \mu \mathrm{m}^{3}\right)$, máxima dimensão linear $(\mathrm{MDL}, \mu \mathrm{m})$ e razão superfície/volume $(\mathrm{S} / \mathrm{V})$ de táxons fitoplanctônicos e ficoperifíticos do Parque Estadual das Fontes do Ipiranga, São Paulo, SP, Brasil. As formas geométricas $(\mathrm{FG})$ correspondentes às siglas da tabela podem ser conferidas na seção Material e métodos (ver tabelas 1 e 2). Os táxons estão ordenados por classe, na seguinte sequência: CYAN: Cyanobacteria, BACI: Bacillariophyceae, CHLO: Chlorophyceae, CHRY: Chrysophyceae, COSC: Coscinodiscophyceae, CRAS: Craspedomonadophyceae, CRYP: Cryptophyceae, DINO: Dinophyceae, EUGL: Euglenophyceae, EUST: Eustigmatophyceae, FRAG: Fragilariophyceae, PRAS: Prasinophyceae, PRYM: Prymnesiophyceae, RAPH: Raphydophyceae, TREB: Trebouxiophyceae, ULVO: Ulvophyceae, XANT: Xanthophyceae, ZYGN: Zygnemaphyceae.

Table 3. Biovolume (BV, $\mu \mathrm{m}^{3}$ ), greatest axial linear dimension (GALD, $\mu \mathrm{m}$ ) and surface/volume ratio (S/V) of phytoplanktonic and phycoperiphytic taxa from Parque Estadual das Fontes do Ipiranga, São Paulo, São Paulo State, Brazil. Geometric forms (GF) corresponding to the abbreviations in the table can be checked in the section Material and methods (tables 1 and 2). The taxa are ordinated by class, in the following sequence: CYAN: Cyanobacteria, BACI: Bacillariophyceae, CHLO: Chlorophyceae, CHRY: Chrysophyceae, COSC: Coscinodiscophyceae, CRAS: Craspedomonadophyceae, CRYP: Cryptophyceae, DINO: Dinophyceae, EUGL: Euglenophyceae, EUST: Eustigmatophyceae, FRAG: Fragilariophyceae, PRAS: Prasinophyceae, PRYM: Prymnesiophyceae, RAPH: Raphydophyceae, TREB: Trebouxiophyceae, ULVO: Ulvophyceae, XANT: Xanthophyceae, ZYGN: Zygnemaphyceae.

\begin{tabular}{|c|c|c|c|c|c|}
\hline Classe & Táxon & FG & MDL & BV & $\mathrm{S} / \mathrm{V}$ \\
\hline CYAN & Aphanizomenon gracile (Lemmermann) Lemmermann & cil & 164,0 & $1.153,8$ & 1,5 \\
\hline CYAN & Aphanocapsa delicatissima W. West \& G.S. West & esf & 47,4 & 41,0 & 5,9 \\
\hline CYAN & Aphanocapsa elachista W. West \& G.S. West & esf & 33,0 & 303,9 & 3,8 \\
\hline CYAN & $\begin{array}{l}\text { Aphanocapsa incerta (Lemmermann) G. Cronberg \& } \\
\text { Komárek }\end{array}$ & esf & 16,0 & $2.065,2$ & 0,4 \\
\hline CYAN & Aphanocapsa koordersii K.Strøm & esf & 70,0 & 782,2 & 2,3 \\
\hline CYAN & Aphanothece clathrata W. West \& G.S. West & esfo & 45,0 & 63,6 & 5,4 \\
\hline CYAN & $\begin{array}{l}\text { Aphanothece smithii Komárková-Legnerová \& G. } \\
\text { Cronberg }\end{array}$ & esfo & 45,0 & 73,2 & 3,8 \\
\hline CYAN & Borzia susedana Ercegovic & cil & 7,0 & 10,9 & 4,1 \\
\hline CYAN & Chroococcus minimus (Keissler) Lemmermann & esf & 6,0 & 109,8 & 1,4 \\
\hline CYAN & Chroococcus minor (Kützing) Nägeli & esf & 4,0 & 27,1 & 1,8 \\
\hline CYAN & $\begin{array}{l}\text { Coelosphaerium evidenter-marginatum M.T.P. Azevedo \& } \\
\text { C.L. Sant'Anna }\end{array}$ & esf & 52,0 & $44.602,3$ & 0,1 \\
\hline CYAN & Coelosphaerium minutissimum Lemmermann & esf & 8,0 & 15,7 & 6,0 \\
\hline CYAN & $\begin{array}{l}\text { Coelosphaerium punctiferum Komárek \& Komárková- } \\
\text { Legnerová }\end{array}$ & $\begin{array}{l}\text { esfext - } \\
\text { esfint* }\end{array}$ & 23,0 & 857,3 & 1,4 \\
\hline CYAN & Cyanonephron styloides Hickel & cil & 6,0 & 25,1 & 4,0 \\
\hline CYAN & $\begin{array}{l}\text { Cylindrospermopsis raciborskii (Woloszynska) Seenayya \& } \\
\text { Subba Raju }\end{array}$ & cil & 126,5 & 751,2 & 1,5 \\
\hline CYAN & $\begin{array}{l}\text { Dolichospermum planctonicum (Brunnthaller) Wacklin, L. } \\
\text { Hoffm. \& Komárek (= Anabaena planctonica } \text { Brunnthaler) }\end{array}$ & cil & 464,2 & $28.686,1$ & 0,5 \\
\hline CYAN & $\begin{array}{l}\text { Epigloeosphaera glebulenta (Zalessky) Komárková- } \\
\text { Legnerová }\end{array}$ & cil & 35,0 & 27,5 & 4,1 \\
\hline CYAN & Geitleribactron subaequale (Geitler) Komárek & cil & 4,3 & 17,8 & 2,3 \\
\hline CYAN & $\begin{array}{l}\text { Geitlerinema amphibium (C. Agardh ex Gomont) } \\
\text { Anagnostidis }\end{array}$ & cil & 39,0 & 116,4 & 3,1 \\
\hline CYAN & Geitlerinema jasorvense (Vouk) Anagnostidis & cil & 378,0 & 931,8 & 2,3 \\
\hline CYAN & Geitlerinema lemmermannii (Woloszynska) Anagnostidis & cil & 94,0 & 295,3 & 2,5 \\
\hline CYAN & Geitlerinema splendidum (Greville ex Gomont) Anagnostidis & cil & 34,5 & 129,7 & 2,3 \\
\hline
\end{tabular}


Tabela 3 (continuação)

\begin{tabular}{|c|c|c|c|c|c|}
\hline Classe & Táxon & FG & MDL & $\mathrm{BV}$ & $\mathrm{S} / \mathrm{V}$ \\
\hline CYAN & $\begin{array}{l}\text { Geitlerinema unigranulatum (R.N.Singh) J.Komárek \& } \\
\text { M.T.P.Azevedo }\end{array}$ & cil & 102,8 & 189,0 & 2,6 \\
\hline CYAN & Johannesbaptistia pellucida (Dickie) W.R. Taylor \& Drouet & esf & 80,0 & $1.047,2$ & 1,2 \\
\hline CYAN & Lemmermanniella pallida (Lemmermann) Geitler & esfo & 13,5 & 91,6 & 7,3 \\
\hline CYAN & $\begin{array}{l}\text { Leptolyngbya angustissima (W. West \& G.S. West) } \\
\text { Anagnostidis \& Komárek }\end{array}$ & cil & 18,5 & 149,7 & 1,9 \\
\hline CYAN & Leptolyngbya elongata (Thomas \& Gonzalves) Anagnostidis & cil & 32,8 & 269,4 & 1,7 \\
\hline CYAN & $\begin{array}{l}\text { Leptolyngbya lagerheimii (Gomont) Anagnostidis \& } \\
\text { Komárek }\end{array}$ & cil & 20,0 & 112,3 & 2,6 \\
\hline CYAN & $\begin{array}{l}\text { Leptolyngbya perelegans (Lemmermann) Anagnostidis \& } \\
\text { Komárek }\end{array}$ & cil & 100,8 & 172,9 & 2,9 \\
\hline CYAN & Leptolyngbya tenuis (Gomont) Anagnostidis \& Komárek & cil & 17,5 & 26,2 & 4,2 \\
\hline CYAN & Merismopedia glauca (Ehrenberg) Kützing & Pret & 11,8 & 145,1 & 1,7 \\
\hline CYAN & Merismopedia punctata Meyen & Pret & 48,0 & 345,6 & 2,2 \\
\hline CYAN & Merismopedia tenuissima Lemmermann & Pret & 11,1 & 78,8 & 2,5 \\
\hline CYAN & Microcystis aeruginosa (Kützing) Kützing $(\mathrm{G})$ & especial & 111,7 & $172.040,0$ & 0,3 \\
\hline CYAN & Microcystis aeruginosa (Kützing) Kützing (M) & especial & 42,1 & $11.353,9$ & 0,5 \\
\hline CYAN & Microcystis aeruginosa (Kützing) Kützing (P) & especial & 27,3 & $2.578,5$ & 1,1 \\
\hline CYAN & $\begin{array}{l}\text { Microcystis panniformis Komárek, Komárková-Legnerová, } \\
\text { C.L. Sant'Anna, M.T.P. Azevedo, \& P.A.C. Senna (P) }\end{array}$ & especial & 49,0 & $37.066,2$ & 0,2 \\
\hline CYAN & $\begin{array}{l}\text { Microcystis panniformis Komárek, Komárková-Legnerová, } \\
\text { C.L. Sant'Anna, M.T.P. Azevedo, \& P.A.C. Senna (M) }\end{array}$ & especial & 77,0 & $120.141,2$ & 0,2 \\
\hline CYAN & $\begin{array}{l}\text { Microcystis panniformis Komárek, Komárková-Legnerová, } \\
\text { C.L. Sant'Anna, M.T.P. Azevedo, \& P.A.C. Senna (G) }\end{array}$ & especial & 168,2 & $543.599,4$ & 0,2 \\
\hline CYAN & Microcystis protocystis Crow & esf & 4,2 & $6.986,0$ & 1,4 \\
\hline CYAN & Myxobaktron hirudiforme G.S. West & esfo & 24,0 & 50,3 & 2,4 \\
\hline CYAN & Oscillatoria pulcherrima M.T.P. Azevedo \& C.L. Sant'Anna & cil & 57,5 & $25.020,9$ & 0,7 \\
\hline CYAN & Oscillatoria sancta Kützing ex Gomont & cil & 200,0 & $12.723,5$ & 0,5 \\
\hline CYAN & $\begin{array}{l}\text { Phormidium chlorinum (Kützing ex Gomont) Umezaki \& } \\
\text { Watanabe }\end{array}$ & cil & 34,1 & 267,0 & 1,8 \\
\hline CYAN & Phormidium hamelii (Frémy) Anagnostidis \& Komárek & cil & 57,0 & 91,4 & 1,4 \\
\hline CYAN & Phormidium natans (Gomont) P.A.C.Senna \& P.Compère & cil & 68,0 & 854,5 & 1,1 \\
\hline CYAN & $\begin{array}{l}\text { Phormidium simplicissimum (Gomont) Anagnostidis \& } \\
\text { Komárek }\end{array}$ & cil & 49,0 & 153,9 & 2,3 \\
\hline CYAN & $\begin{array}{l}\text { Planktolyngbya contorta (Lemmermann) Anagnostidis \& } \\
\text { Komárek }\end{array}$ & cil & 24,0 & 27,1 & 4,0 \\
\hline CYAN & Planktothrix agardhii (Gomont) Anagnostidis \& Komárek & cil & 99,6 & $1.903,5$ & 1,1 \\
\hline CYAN & Pseudanabaena galeata Böcher & cil & 87,9 & 630,5 & 2,0 \\
\hline CYAN & Pseudanabaena limnetica (Lemmermann) Komárek & cil & 42,0 & 47,5 & 4,3 \\
\hline CYAN & $\begin{array}{l}\text { Pseudanabaena mucicola (Naumann \& Huber-Pestalozzi) } \\
\text { Schwabe }\end{array}$ & cil & 10,0 & 17,0 & 2,9 \\
\hline CYAN & Radiocystis fernandoi Komárek \& Komárková-Legnerová & esf & 40,0 & $9.382,9$ & 0,8 \\
\hline CYAN & Rhabdoderma sancti-pauli M.T.P. Azevedo et al. & esfo & 62,5 & 704,7 & 4,2 \\
\hline CYAN & Rhabdoderma sigmoideum Moore \& Carter & cil & 30,0 & 27,1 & 3,8 \\
\hline
\end{tabular}


Tabela 3 (continuação)

\begin{tabular}{|c|c|c|c|c|c|}
\hline Classe & Táxon & FG & MDL & $\mathrm{BV}$ & $\mathrm{S} / \mathrm{V}$ \\
\hline$\overline{\text { CYAN }}$ & Romeria gracilis (Koczwara) Koczwara & cil & 6,7 & 170,5 & 2,5 \\
\hline CYAN & $\begin{array}{l}\text { Sphaerocavum brasiliense M.T.P. Azevedo \& C.L. } \\
\text { Sant'Anna } P\end{array}$ & especial & 26,5 & $2.690,1$ & 0,7 \\
\hline CYAN & $\begin{array}{l}\text { Sphaerocavum brasiliense M.T.P. Azevedo \& C.L. } \\
\text { Sant'Anna } M\end{array}$ & especial & 69,6 & $14.162,7$ & 0,6 \\
\hline CYAN & $\begin{array}{l}\text { Sphaerocavum brasiliense M.T.P. Azevedo \& C.L. } \\
\text { Sant'Anna } G\end{array}$ & especial & 120,2 & $38.694,9$ & 0,6 \\
\hline CYAN & $\begin{array}{l}\text { Sphaerocavum brasiliense M.T.P. Azevedo \& C.L. } \\
\text { Sant'Anna } G G\end{array}$ & especial & 314,5 & $243.530,2$ & 0,6 \\
\hline CYAN & Synechococcus nidulans (Pringsheim) Komárek & cil & 3,7 & 6,2 & 4,1 \\
\hline CYAN & Synechocystis aquatilis Sauvageau & esf & 4,0 & 32,7 & 1,5 \\
\hline CYAN & Tychonema bornetii (Zukal) Anagnostidis \& Komárek & cil & 23,7 & 383,5 & 1,3 \\
\hline $\mathrm{BACI}$ & Achnanthidium catenatum (Bily \& Marvan) Lange-Bertalot & Peli & 13,7 & 86,3 & 0,5 \\
\hline BACI & Achnanthidium exiguum (Grunow) Czarnecki & Peli & 11,0 & 129,6 & 2,5 \\
\hline $\mathrm{BACI}$ & Achnanthidium microcephalum Kützing & Peli & 19,3 & 200,5 & 1,7 \\
\hline $\mathrm{BACI}$ & Achnanthidium minutissimum (Kützing) Czarnecki & Peli & 19,5 & 134,0 & 2,1 \\
\hline BACI & $\begin{array}{l}\text { Achnanthidium saprophilum (Kobayasi \& Mayama) } \\
\text { Round \& Bukhtiyarova }\end{array}$ & Peli & 13,8 & 153,2 & 2,7 \\
\hline $\mathrm{BACI}$ & Actinella brasiliensis Grunow & Pfoi & 52,0 & $1.129,6$ & 1,5 \\
\hline BACI & $\begin{array}{l}\text { Amphora copulata (Kützing) Schoeman \& R.E.M. } \\
\text { Archibald }\end{array}$ & Cimb & 41,0 & 975,7 & 0,3 \\
\hline BACI & Brachysira brebissonii Ross & Peli & 27,4 & 496,5 & 2,1 \\
\hline $\mathrm{BACI}$ & Brachysira vitrea (Grunow) Ross & Peli & 21,7 & 474,4 & 1,6 \\
\hline $\mathrm{BACI}$ & Cymbella affinis Kützing & P0.5elip & 23,5 & 364,0 & 1,0 \\
\hline BACI & $\begin{array}{l}\text { Cymbopleura naviculiformis (Auerswald ex Heiberg) } \\
\text { Krammer }\end{array}$ & P0.5elip & 21,5 & 391,1 & 0,6 \\
\hline $\mathrm{BACI}$ & Encyonema lunatum (W. Smith) van Heurck & Cimb & 35,1 & 404,1 & 0,7 \\
\hline $\mathrm{BACI}$ & Encyonema mesianum (Cholnoky) Mann & Cimb & 34,0 & 640,9 & 0,6 \\
\hline $\mathrm{BACI}$ & Encyonema minutum (Hilse) Mann & $\mathrm{Cimb}$ & 15,1 & 129,6 & 0,8 \\
\hline $\mathrm{BACI}$ & Encyonema neogracile Krammer & $\mathrm{Cimb}$ & 40,8 & 492,7 & 0,7 \\
\hline $\mathrm{BACI}$ & Encyonema silesiacum (Bleisch) Mann & Cimb & 24,0 & 745,7 & 0,5 \\
\hline $\mathrm{BACI}$ & Encyonopsis raytonensis (Cholnoky) Krammer & Cimb & 30,0 & 478,4 & 0,6 \\
\hline $\mathrm{BACI}$ & Eolimna minima (Grunow) Lange-Bertalot & Peli & 10,8 & 126,2 & 2,4 \\
\hline $\mathrm{BACI}$ & Eunotia bilunaris (Ehrenberg) Mills morfotipo 1 & Pfoi & 37,0 & 197,5 & 1,1 \\
\hline $\mathrm{BACI}$ & Eunotia bilunaris (Ehrenberg) Mills morfotipo 2 & Pfoi & 166,0 & 959,8 & 1,0 \\
\hline $\mathrm{BACI}$ & Eunotia carolina Patrick & Pfoi & 51,0 & $4.806,6$ & 0,3 \\
\hline $\mathrm{BACI}$ & Eunotia exigua (Brébisson ex Kützing) Rabenhorst & Pfoi & 23,0 & 166,2 & 1,1 \\
\hline $\mathrm{BACI}$ & Eunotia faba (Ehrenberg) Grunow & Pfoi & 9,0 & 320,4 & 0,8 \\
\hline $\mathrm{BACI}$ & Eunotia georgii Metzeltin \& Lange-Bertalot & Pfoi & 44,0 & $4.838,1$ & 0,4 \\
\hline $\mathrm{BACI}$ & Eunotia incisa Smith ex Gregory & Pfoi & 21,0 & 815,2 & 0,7 \\
\hline $\mathrm{BACI}$ & Eunotia indica Grunow & Pfoi & 49,4 & $1.769,2$ & 0,4 \\
\hline $\mathrm{BACI}$ & Eunotia intermedia (Krasske) Nörpel \& Lange-Bertalot & Pfoi & 10,0 & 255,3 & 0,5 \\
\hline $\mathrm{BACI}$ & Eunotia minor (Kützing) Grunow in van Heurck & Pfoi & 45,0 & $1.979,2$ & 0,4 \\
\hline
\end{tabular}


Tabela 3 (continuação)

\begin{tabular}{|c|c|c|c|c|c|}
\hline Classe & Táxon & FG & MDL & BV & $\mathrm{S} / \mathrm{V}$ \\
\hline BACI & Eunotia muscicola var. tridentula Nörpel \& Lange-Bertalot & Pfoi & 17,0 & 173,1 & 0,8 \\
\hline BACI & Eunotia pileus Ehrenberg & Pfoi & 85,2 & $23.554,5$ & 0,2 \\
\hline BACI & Eunotia praerupta Ehrenberg & Pfoi & 45,6 & $2.085,1$ & 0,4 \\
\hline BACI & Eunotia rhomboidea Hustedt & Pfoi & 16,3 & 242,8 & 0,7 \\
\hline BACI & Eunotia subarcuatoides Alles, Nörpel \& Lange-Bertalot & Pfoi & 10,1 & 87,3 & 1,0 \\
\hline BACI & Eunotia sudetica Müller & Pfoi & 38,0 & $3.407,4$ & 0,5 \\
\hline BACI & Eunotia tenella (Grunow) Hustedt & Pfoi & 16,0 & 339,3 & 0,9 \\
\hline BACI & Eunotia zygodon Ehrenberg & Pfoi & 68,8 & $12.806,4$ & 0,2 \\
\hline BACI & $\begin{array}{l}\text { Frustulia crassinervia (Brébisson) Lange-Bertalot } \\
\text { \& Krammer }\end{array}$ & Peli & 44,4 & $2.216,0$ & 0,5 \\
\hline BACI & Frustulia rhomboides (Ehrenberg) De Toni & Peli & 69,0 & $4.431,6$ & 0,3 \\
\hline BACI & Frustulia saxonica Rabenhorst & Peli & 40,4 & $1.808,4$ & 1,2 \\
\hline BACI & Gomphonema affine Kützing var. affine & Gomph & 44,0 & $5.970,7$ & 0,4 \\
\hline BACI & Gomphonema affine var. rhombicum Reichardt & Gomph & 67,0 & $21.651,3$ & 0,2 \\
\hline BACI & Gomphonema angustatum (Kützing) Rabenhorst & Gomph & 28,0 & $1.365,4$ & 0,2 \\
\hline BACI & Gomphonema apicatum Ehrenberg & Gomph & 35,8 & $4.514,1$ & 0,4 \\
\hline BACI & $\begin{array}{l}\text { Gomphonema augur var. turris (Ehrenberg) Lange- } \\
\text { Bertalot in Krammer \& Lange-Bertalot }\end{array}$ & Gomph & 43,0 & $7.552,0$ & 0,3 \\
\hline BACI & Gomphonema gracile Ehrenberg & Gomph & 63,0 & $4.484,5$ & 0,5 \\
\hline BACI & Gomphonema lagenula Kützing & Gomph & 23,0 & 622,4 & 0,9 \\
\hline BACI & Gomphonema laticollum Reichardt & Gomph & 46,0 & $33.039,4$ & 0,1 \\
\hline BACI & Gomphonema parvulum (Kützing) Kützing & Gomph & 27,0 & $1.096,8$ & 0,6 \\
\hline BACI & Gomphonema pseudoaugur Lange-Bertalot & Gomph & 24,0 & $1.190,7$ & 0,5 \\
\hline BACI & $\begin{array}{l}\text { Gomphonema pumilum var. rigidum Reichardt \& } \\
\text { Lange-Bertalot }\end{array}$ & Gomph & 24,0 & 692,5 & 0,8 \\
\hline BACI & Gomphonema turris var. brasiliense (Fricke) Frenguelli & Gomph & 41,0 & $3.394,9$ & 0,5 \\
\hline BACI & Gomphonema venusta Passy, Kociolek \& Lowe & Gomph & 33,5 & 749,6 & 1,7 \\
\hline BACI & Navicula cryptocephala Kützing & Peli & 30,0 & 589,1 & 1,7 \\
\hline BACI & Navicula cryptotenella Lange-Bertalot & Peli & 30,0 & 518,4 & 1,8 \\
\hline BACI & Navicula erifuga Lange-Bertalot & Peli & 19,0 & 358,1 & 1,9 \\
\hline BACI & Navicula notha Wallace & Peli & 20,0 & 171,5 & 2,9 \\
\hline BACI & Nitzschia acicularis (Kützing) W. Smith & Plos & 92,3 & $2.041,3$ & 0,5 \\
\hline BACI & Nitzschia amphibia Grunow & Plos & 17,0 & 420,8 & 0,3 \\
\hline $\mathrm{BACI}$ & Nitzschia graciliformis Lange-Bertalot \& Simonsen & Plos & 200,0 & $3.850,0$ & 0,4 \\
\hline BACI & Nitzschia gracilis Hantzsch ex Rabenhorst & Plos & 105,0 & 949,4 & 0,4 \\
\hline BACI & Nitzschia linearis (Agardh) W. Smith & Plos & 90,0 & $1.535,6$ & 0,3 \\
\hline BACI & Nitzschia palea (Kützing) W. Smith & Plos & 24,8 & 214,8 & 1,0 \\
\hline BACI & Nitzschia paleaformis Hustedt & Plos & 26,0 & 82,8 & 0,9 \\
\hline BACI & Nitzschia sigmoidea (Nitzsch) W. Smith & Plos & 146,0 & $6.860,0$ & 0,3 \\
\hline BACI & Pinnularia biceps Gregory var. biceps & Pret & 72,5 & $5.407,5$ & 0,5 \\
\hline BACI & Pinnularia brauniana (Grunow) F.W. Mills & Pret & 52,0 & $1.872,0$ & 0,8 \\
\hline BACI & Pinnularia divergens var. malayensis Hustedt & Peli & 150,0 & $58.905,0$ & 0,4 \\
\hline BACI & $\begin{array}{l}\text { Pinnularia divergens var. mesoleptiformis Krammer } \\
\text { \& Metzeltin }\end{array}$ & Pret & 89,0 & $16.198,0$ & 0,3 \\
\hline
\end{tabular}


Tabela 3 (continuação)

\begin{tabular}{|c|c|c|c|c|c|}
\hline Classe & Táxon & FG & MDL & BV & $\mathrm{S} / \mathrm{V}$ \\
\hline $\mathrm{BACI}$ & Pinnularia divergens var. sublinearis Cleve & Peli & 88,0 & $22.324,2$ & 0,5 \\
\hline BACI & Pinnularia divergens $\mathrm{W}$. Smith var. divergens & Peli & 62,0 & $8.278,1$ & 0,7 \\
\hline BACI & Pinnularia gentilis (Donkin) Cleve & Pret & 168,0 & $105.000,0$ & 0,2 \\
\hline BACI & Pinnularia gibba var. subundulata (Mayer) Frenguelli & Pret & 67,0 & $3.333,8$ & 0,6 \\
\hline BACI & Pinnularia mesogongyla Cleve & Pret & 62,5 & $5.361,8$ & 0,5 \\
\hline BACI & Pinnularia nobilis (Ehrenberg) Ehrenberg & Pret & 200,0 & $203.700,0$ & 0,1 \\
\hline BACI & Pinnularia romanorum Metzeltin \& Lange-Bertalot & Pret & 128,0 & $45.720,0$ & 0,2 \\
\hline BACI & Pinnularia subgibba var. lanceolata Gaiser \& Johansen & Pret & 56,0 & $6.435,0$ & 0,4 \\
\hline BACI & Pinnularia viridiformis Krammer & Pret & 76,0 & $24.320,0$ & 0,3 \\
\hline BACI & Pinnularia viridis (Nitzsch) Ehrenberg & Peli & 150,0 & $47.124,0$ & 0,2 \\
\hline BACI & $\begin{array}{l}\text { Planothidium lanceolatum (Brébisson ex Kützing) } \\
\text { Lange-Bertalot }\end{array}$ & Peli & 20,0 & 285,9 & 1,9 \\
\hline BACI & Planothidium rostratum (Oestrup) Lange-Bertalot & Peli & 12,0 & 188,5 & 2,1 \\
\hline BACI & Sellaphora pupula (Kützing) Mereschkovsky & Peli & 48,0 & $4.913,7$ & 0,8 \\
\hline BACI & Stauroneis phoenicenteron (Nitzsch) Ehrenberg & Peli & 169,5 & $122.649,6$ & 2,1 \\
\hline BACI & $\begin{array}{l}\text { Stenopterobia delicatissima (Lewis) Brébisson ex } \\
\text { van Heurck }\end{array}$ & Plos & 58,6 & $2.051,0$ & 0,3 \\
\hline BACI & Surirella guatimalensis Ehrenberg & Peli & 145,0 & $34.899,4$ & 1,0 \\
\hline BACI & Surirella linearis var. constricta Grunow & Peli & 227,5 & $153.343,8$ & 0,3 \\
\hline CHLO & $\begin{array}{l}\text { Acutodesmus acuminatus (Lagerheim) Tsarenko) } \\
\quad(=\text { Scenedesmus acuminatus (Lagerheim) Chodat) }\end{array}$ & cil +2 cone & 16,4 & 375,5 & 1,7 \\
\hline CHLO & $\begin{array}{l}\text { Acutodesmus obliquus (Turpin) E. Hegewald \& } \\
\text { Hanagata (= Scenedesmus obliquus (Turpin) Kützing) }\end{array}$ & esfo & 12,5 & 551,2 & 1,2 \\
\hline CHLO & Ankistrodesmus bernardii Komárek & cil +2 cone & 50,0 & 813,8 & 2,8 \\
\hline CHLO & Ankistrodesmus densus Koršikov & mon & 43,0 & $2.026,3$ & 1,0 \\
\hline CHLO & Ankistrodesmus falcatus (Corda) Ralfs & cil +2 cone & 40,0 & 538,7 & 2,6 \\
\hline CHLO & Ankistrodesmus fusiformis Corda ex Koršikov & cil +2 cone & 41,0 & 748,5 & 2,3 \\
\hline CHLO & Ankistrodesmus gracilis (Reinsch) Koršikov & mon & 37,0 & 353,8 & 2,5 \\
\hline CHLO & Ankistrodesmus spiralis (W.B. Turner) Lemmermann & cil +2 cone & 41,7 & 402,7 & 3,5 \\
\hline CHLO & Carteria multifilis (Fresenius) O. Dill & esf & 13,0 & $1.012,8$ & 0,5 \\
\hline CHLO & Carteria platyrhyncha Ettl & esfo & 9,1 & 247,0 & 0,8 \\
\hline CHLO & Carteria sanpaulensis Skvortzov ex C. Bicudo & esf & 13,0 & $1.150,3$ & 0,5 \\
\hline CHLO & Chaetosphaeridium globosum (Nordstedt) Klebahn & esf & 13,0 & 678,1 & 0,6 \\
\hline CHLO & Characium acuminatum Braun & elip & 12,7 & 195,5 & 0,9 \\
\hline CHLO & Characium rostratum Reinhard ex Printz & elip & 16,3 & 219,1 & 0,8 \\
\hline CHLO & Characium westianum Printz & esfo & 35,0 & 458,2 & 1,0 \\
\hline CHLO & Chlamydomonas agloeformis Pascher & esfo & 13,1 & 264,2 & 0,8 \\
\hline CHLO & Chlamydomonas debaryana Goroschankin & esfo & 10,5 & 303,0 & 0,7 \\
\hline CHLO & Chlamydomonas epibiotica Ettl & esfo & 7,1 & 96,1 & 1,1 \\
\hline CHLO & Chlamydomonas gleopara Rodhe \& Skuja & esfo & 8,5 & 95,2 & 1,2 \\
\hline CHLO & Chlamydomonas planctogloea Skuja & esf & 3,7 & 19,2 & 2,0 \\
\hline CHLO & Chlamydomonas sagittula Skuja & esfo & 7,0 & 14,7 & 3,0 \\
\hline CHLO & Chlamydomonas sordida Ettl & esfo & 6,6 & 27,3 & 2,2 \\
\hline CHLO & Chlorococcum infusionum (Schrank) Meneghini & esf & 10,5 & 601,0 & 0,6 \\
\hline CHLO & Chlorogonium fusiforme Matvienko & 2 cone & 20,0 & 64,1 & 34,6 \\
\hline
\end{tabular}


Tabela 3 (continuação)

\begin{tabular}{|c|c|c|c|c|c|}
\hline Classe & Táxon & FG & MDL & BV & $\mathrm{S} / \mathrm{V}$ \\
\hline CHLO & Chlorolobion braunii (Nägeli) Komárek & 2 cone & 14,5 & 103,6 & 17,1 \\
\hline CHLO & Chloromonas frigida Gerloff \& Ettl & esfo & 7,8 & 119,2 & 1,0 \\
\hline CHLO & Chloromonas pumilio Ettl & esfo & 6,7 & 14,2 & 2,4 \\
\hline CHLO & Choricystis chodatii (Jaag) Fott & esfo & 10,0 & 47,1 & 1,6 \\
\hline CHLO & Chorycistis minor (Skuja) Fott & esfo & 7,3 & 31,3 & 1,8 \\
\hline CHLO & Closteriopsis acicularis (Chodat) J.H. Belcher \& Swale & cil + 2cone & 106,4 & $1.347,7$ & 1,7 \\
\hline CHLO & Closteriopsis longissima (Lemmermann) Lemmermann & cil +2 cone & 38,0 & 95,5 & 2,5 \\
\hline CHLO & Coccomonas orbicularis F. Stein & esf & 6,6 & 98,9 & 1,1 \\
\hline CHLO & Coelastrum astroideum De Notaris & cone & 17,3 & 427,9 & 1,8 \\
\hline CHLO & Coelastrum microporum Nägeli & esf & 18,5 & $1.344,9$ & 1,0 \\
\hline CHLO & Coelastrum proboscideum Bohlin & cone & 18,0 & $1.032,0$ & 1,4 \\
\hline CHLO & $\begin{array}{l}\text { Coelastrum pulchrum Schmidle (= Coelastrum } \\
\text { cambricum } \mathrm{W} \text {. Archer) }\end{array}$ & esf & 14,0 & 278,3 & 1,5 \\
\hline CHLO & Coelastrum reticulatum (P.A. Dangeard) Senn & esf & 25,5 & $8.722,9$ & 0,2 \\
\hline CHLO & Coenochloris hindakii Komárek & esf & 30,0 & 696,9 & 1,1 \\
\hline CHLO & Coenochloris planoconvexa Hindák & esfo & 30,0 & 266,0 & 1,2 \\
\hline CHLO & Coenocystis micrococca Komárek & esf & 2,3 & 6,4 & 2,6 \\
\hline CHLO & Desmatractum indutum (Geitler) Pascher & esfo & 45,0 & $2.453,4$ & 0,5 \\
\hline CHLO & $\begin{array}{l}\text { Desmodesmus armatus (R. Chodat) E. Hegewald } \\
\quad(=\text { Scenedesmus armatus (R. Chodat) R. Chodat) }\end{array}$ & esfo & 11,0 & 387,3 & 1,2 \\
\hline CHLO & $\begin{array}{l}\text { Desmodesmus denticulatus (Lagerheim) } \\
\quad(=\text { Scenedesmus denticulatus Lagerheim) }\end{array}$ & esfo & 11,4 & 414,5 & 1,2 \\
\hline CHLO & $\begin{array}{l}\text { Desmodesmus dispar (Brébisson) E. Hegewald } \\
\text { (= Scenedesmus dispar Brébisson) }\end{array}$ & esfo & 8,6 & 246,6 & 1,4 \\
\hline CHLO & $\begin{array}{l}\text { Desmodesmus granulatus }(\mathrm{W} . \text { West \& G.S. West) Hentschke } \\
\text { \& Torgan (= Scenedesmus granulatus W. West \& G.S. West })\end{array}$ & esfo & 8,5 & 80,1 & 1,6 \\
\hline CHLO & $\begin{array}{l}\text { Desmodesmus intermedius (Chodat) E. Hegewald } \\
\text { (= Scenedesmus intermedius Chodat) }\end{array}$ & esfo & 18,0 & 80,1 & 2,1 \\
\hline CHLO & $\begin{array}{l}\text { Desmodesmus lefevrei (Deflandre) S.S. An, T. Friedl } \\
\text { \& E. Hegewald (= Scenedesmus lefevrei Deflandre) }\end{array}$ & esfo & 19,0 & 279,8 & 1,3 \\
\hline CHLO & Desmodesmus maximus (W. West \& G.S.West) E. Hegewald & esfo & 14,0 & $1.194,6$ & 0,8 \\
\hline CHLO & $\begin{array}{l}\text { Desmodesmus opoliensis (P.G. Richter) E. Hegewald } \\
\quad(=\text { Scenedesmus opoliensis P.G. Richter) }\end{array}$ & esfo & 25,1 & 238,1 & 1,6 \\
\hline CHLO & $\begin{array}{l}\text { Desmodesmus opoliensis var. mononensis (R. Chodat) } \\
\text { E. Hegewald }\end{array}$ & esfo & 18,0 & 461,8 & 0,7 \\
\hline CHLO & Desmodesmus polyspinosus (T. Hortobágyi) E. Hegewald & esfo & 9,0 & 150,8 & 1,3 \\
\hline CHLO & $\begin{array}{l}\text { Desmodesmus pseudodenticulatus (E. Hegewald) E. Hegewald } \\
\quad \text { (= Scenedesmus pseudodenticulatus } \text { E. Hegewald) }\end{array}$ & esfo & 9,0 & 104,7 & 2,0 \\
\hline CHLO & $\begin{array}{l}\text { Desmodesmus serratus (Corda) S.S. An, T. Friedl \& } \\
\text { E. Hegewald) (= Scenedesmus serratus Corda) }\end{array}$ & esfo & 13,8 & $1.290,0$ & 0,9 \\
\hline CHLO & $\begin{array}{l}\text { Desmodesmus spinosus (Chodat) E. Hegewald) } \\
\text { (= Scenedesmus spinosus Chodat) }\end{array}$ & esfo & 15,4 & 111,9 & 1,8 \\
\hline CHLO & Dimorphococcus lunatus A. Braum & esfo & 5,0 & 23,6 & 1,8 \\
\hline CHLO & Diplochloris decussata Koršikov & esfo & 50,0 & 212,8 & 2,2 \\
\hline CHLO & Elakatothrix gelatinosa Wille & cone & 21,7 & 25,8 & 6,6 \\
\hline
\end{tabular}


Tabela 3 (continuação)

\begin{tabular}{|c|c|c|c|c|c|}
\hline Classe & Táxon & FG & MDL & $\mathrm{BV}$ & $\mathrm{S} / \mathrm{V}$ \\
\hline CHLO & $\begin{array}{l}\text { Enallax acutiformis (B. Schröder) F. Hindák } \\
\quad(=\text { Scenedesmus acutiformis B. Schröder }\end{array}$ & esfo & 14,1 & 602,0 & 1,3 \\
\hline CHLO & Eutetramorus fottii (Hindák) Komárek & esf & 50,0 & $2.144,7$ & 0,8 \\
\hline CHLO & Eutetramorus globosus Walton & esf & 50,8 & 13,6 & 3,9 \\
\hline CHLO & Fusola viridis J. Snow & elip & 12,5 & 32,7 & 1,6 \\
\hline CHLO & Golenkinia radiata Chodat & esf & 27,0 & 179,6 & 0,9 \\
\hline CHLO & Kirchneriella aperta Teiling & mon & 10,0 & 380,9 & 1,2 \\
\hline $\mathrm{CHLO}$ & $\begin{array}{l}\text { Kirchneriella contorta (Schmidle) Bohlin } \\
\quad(=\text { Pseudokirchneriella contorta (Schmidle) F. Hindák) }\end{array}$ & mon & 5,2 & 16,4 & 1,3 \\
\hline CHLO & Kirchneriella contorta var. elegans (Playfair) Komárek & mon & 30,0 & 399,9 & 1,0 \\
\hline CHLO & Kirchneriella dianae (Bohlin) C. Gonzalez & mon & 20,0 & 404,7 & 1,6 \\
\hline CHLO & Kirchneriella irregularis (G.M. Smith) Koršikov & mon & 20,0 & 162,4 & 1,8 \\
\hline CHLO & Kirchneriella lunaris (Kirchner) K. Möbius & mon & 35,0 & 177,6 & 2,0 \\
\hline CHLO & Kirchneriella microscopica Nygaard & mon & 10,0 & 37,3 & 2,5 \\
\hline CHLO & Kirchneriella obesa (W. West) W. West \& G.S. West & mon & 30,0 & 423,2 & 1,1 \\
\hline CHLO & Kirchneriella pinguis Hindák & mon & 10,0 & 11,4 & 4,2 \\
\hline CHLO & Lagerheimia ciliata (Lagerheim) Chodat & esfo & 36,0 & 210,7 & 1,2 \\
\hline CHLO & Micractinium pusillum Fresenius & esf & 48,8 & $1.534,3$ & 0,4 \\
\hline CHLO & Monoraphidium arcuatum (Koršikov) Hindák & mon & 41,4 & 104,3 & 3,2 \\
\hline CHLO & Monoraphidium circinale (Nygaard) Nygaard & mon & 10,5 & 117,4 & 2,7 \\
\hline CHLO & $\begin{array}{l}\text { Monoraphidium contortum (Thuret) Komàrková- } \\
\text { Legnerová }\end{array}$ & cil +2 cone* & 19,3 & 26,5 & 3,7 \\
\hline CHLO & $\begin{array}{l}\text { Monoraphidium dybowskii (Woloszynska) Hindák \& } \\
\text { Komárkova Legnerová }\end{array}$ & 2 cone & 10,5 & 34,4 & 1,2 \\
\hline CHLO & Monoraphidium flexuosum Komárek & mon & 18,0 & 36,8 & 2,9 \\
\hline CHLO & $\begin{array}{l}\text { Monoraphidium griffithii (Berkeley) Komárková- } \\
\text { Legnerová }\end{array}$ & cil + 2cone & 51,3 & 163,1 & 2,6 \\
\hline CHLO & $\begin{array}{l}\text { Monoraphidium irregulare (G.M. Smith) } \\
\text { Komárková-Legnerová }\end{array}$ & cil +2 cone $*$ & 63,4 & 219,2 & 2,4 \\
\hline CHLO & Monoraphidium komarkovae Nygaard & cil +2 cone & 117,4 & 215,7 & 2,9 \\
\hline CHLO & Monoraphidium minutum (Nägeli) Komárková-Legnerová & mon & 7,8 & 92,3 & 1,2 \\
\hline CHLO & Monoraphidium nanum (Ettl) Hindák & mon & 5,6 & 27,5 & 1,7 \\
\hline CHLO & Monoraphidium pseudobraunii (Belcher \& Swale) Heynig & esfo & 19,4 & 32,7 & 2,9 \\
\hline CHLO & Monoraphidium pusillum (Printz) Komárková-Legnorová & mon & 11,7 & 46,2 & 2,1 \\
\hline CHLO & $\begin{array}{l}\text { Monoraphidium tortile (W. West \& G.S. West) } \\
\text { Komárková-Legnerová }\end{array}$ & cil +2 cone & 17,0 & 31,5 & 3,2 \\
\hline CHLO & $\begin{array}{l}\text { Pectinodesmus regularis (Svirenko) E. Hegewald, M. Wolf, Al. } \\
\text { Keller, Friedl \& Krienitz (= Scenedesmus regularis Svirenko) }\end{array}$ & esfo & 19,7 & 670,2 & 1,2 \\
\hline CHLO & Pediastrum duplex Meyen & cil & 47,7 & $1.789,3$ & 2,1 \\
\hline CHLO & Pediastrum simplex Meyen & cil & 63,0 & $3.117,3$ & 2,1 \\
\hline CHLO & Protoderma viride Kützing & cil & 141,0 & $6.450,1$ & 1,5 \\
\hline CHLO & Pseudodidymocystis fina (Komárek) E. Hegewald \& Deason & elip & 5,2 & 27,5 & 1,8 \\
\hline CHLO & $\begin{array}{l}\text { Pseudodidymocystis planctonica (Koršikov) E.Hegewald } \\
\quad \text { \& Deason }\end{array}$ & elip & 10,0 & 206,2 & 0,8 \\
\hline CHLO & $\begin{array}{l}\text { Pseudoschroederia antillarum (Komárek) E. Hegewald } \\
\text { \& Schnepf }\end{array}$ & 2 cone & 10,0 & 11,5 & 29,2 \\
\hline
\end{tabular}


Tabela 3 (continuação)

\begin{tabular}{|c|c|c|c|c|c|}
\hline Classe & Táxon & FG & MDL & BV & $\mathrm{S} / \mathrm{V}$ \\
\hline CHLO & Quadrigula chodatii (Tanner-Füllemann) G.M. Smith & cil + 2cone & 30,0 & $1.193,0$ & 1,2 \\
\hline CHLO & $\begin{array}{l}\text { Raphidocelis contorta (Schmidle) Marvan, Komárek \& } \\
\text { Comas (= Pseudokirchneriella contorta (Schmidle) Hindák) }\end{array}$ & mon & 8,5 & 15,6 & 2,9 \\
\hline CHLO & Raphidocelis sigmoidea Hindák & mon & 9,9 & $1.531,3$ & 0,8 \\
\hline CHLO & $\begin{array}{l}\text { Raphidocelis subcapitata (Koršikov) G. Nygaard, J. } \\
\text { Komárek, J. Krisitiansen \& O.M. Skulberg }\end{array}$ & mon & 5,0 & 10,5 & 1,6 \\
\hline CHLO & Scenedesmus aculeolatus Reinsch & esfo & 13,0 & 354,7 & 1,2 \\
\hline CHLO & Scenedesmus acutus Meyen & esfo & 26,6 & $1.267,5$ & 0,9 \\
\hline CHLO & Scenedesmus antennatus Brébisson & esfo & 27,6 & 282,7 & 1,6 \\
\hline CHLO & Scenedesmus arcuatus Lemmermann & esfo & 11,0 & 360,0 & 1,2 \\
\hline CHLO & Scenedesmus bernardii G.M. Smith & cil + 2cone & 22,0 & 839,7 & 1,1 \\
\hline CHLO & Scenedesmus bicaudatus (Hansgirg) Chodat & esfo & 22,0 & 191,6 & 1,1 \\
\hline CHLO & Scenedesmus brevispina (G.M. Smith) R. Chodat & esfo & 11,3 & 163,4 & 1,5 \\
\hline CHLO & $\begin{array}{l}\text { Scenedesmus communis E. Hegewald } \\
\quad \text { (= Scenedesmus quadricauda Chodat) }\end{array}$ & esfo & 26,4 & 254,0 & 1,2 \\
\hline CHLO & Scenedesmus dimorphus (Turpin) Kützing & esfo & 13,0 & 435,6 & 1,2 \\
\hline CHLO & Scenedesmus disciformis (Chodat) Fott \& Komárek & esfo & 8,6 & 357,0 & 1,2 \\
\hline CHLO & Scenedesmus ecornis var. disciformis (Chodat) Chodat & esfo & 18,0 & 638,3 & 1,1 \\
\hline CHLO & Scenedesmus ecornis var. ecornis (Ehrenberg) Chodat & esfo & 9,5 & 170,1 & 1,5 \\
\hline CHLO & Scenedesmus gutwinskii Chodat & esfo & 9,0 & 169,6 & 1,6 \\
\hline CHLO & Scenedesmus gutwinskii var. bacsensis Uherkovich & esfo & 22,0 & 117,8 & 1,9 \\
\hline CHLO & Scenedesmus gutwinskii var. heterospina Bodrogsközy & esfo & 12,0 & 84,8 & 1,6 \\
\hline CHLO & Scenedesmus indicus Philipose & esfo & 13,2 & 172,8 & 1,9 \\
\hline CHLO & Scenedesmus insignis (W. West \& G.S. West) Chodat & esfo & 12,0 & 56,5 & 1,6 \\
\hline CHLO & Scenedesmus kissii Hortobágyi & esfo & 10,7 & 414,4 & 1,2 \\
\hline CHLO & Scenedesmus lineares Komárek & esfo & 8,3 & 101,4 & 1,7 \\
\hline CHLO & Scenedesmus longispina $\mathrm{R}$. Chodat & esfo & 15,0 & 282,7 & 1,6 \\
\hline CHLO & Scenedesmus obtusus var. obtusus Meyen & esfo & 8,3 & 447,3 & 1,1 \\
\hline CHLO & Scenedesmus opoliensis var. bicaudatus Hortobágyi & esfo & 22,0 & 323,7 & 1,3 \\
\hline CHLO & Scenedesmus ovalternus Chodat & esfo & 16,0 & $1.387,8$ & 0,8 \\
\hline CHLO & Scenedesmus producto-capitatus Schmula & esfo & 11,0 & 243,3 & 1,5 \\
\hline CHLO & Scenedesmus protuberans F.E. Fritsch \& M.F. Rich & esfo & 36,9 & 661,5 & 1,1 \\
\hline CHLO & Scenedesmus semicristatus Uherkovich & esfo & 10,7 & 414,4 & 1,2 \\
\hline CHLO & Scenedesmus semipulcher Hortobágyi & esfo & 17,9 & 345,3 & 1,2 \\
\hline CHLO & Scenedesmus sempervirens Chodat & esfo & 12,0 & 41,9 & 2,5 \\
\hline CHLO & Scenedesmus setiferus Chodat & esfo & 12,2 & 39,8 & 1,9 \\
\hline CHLO & Scenedesmus smithii Chodat & esfo & 12,0 & 201,1 & 1,3 \\
\hline CHLO & Scenedesmus verrucosus P. González & esfo & 7,0 & 117,3 & 1,3 \\
\hline CHLO & Scenedesmus westii (G.M. Smith) Chodat & esfo & 19,0 & $1.055,6$ & 0,8 \\
\hline CHLO & Schroderia nitzschioides (G.S. West) Koršikov & 2cone & 101,0 & 951,9 & 101,1 \\
\hline CHLO & Schroderia setigera (Schröder) Lemmermann & 2 cone & 100,0 & 654,5 & 120,1 \\
\hline CHLO & Schroderia spiralis (Printz) Koršikov & 2cone & 20,0 & 130,9 & 24,3 \\
\hline
\end{tabular}


Tabela 3 (continuação)

\begin{tabular}{|c|c|c|c|c|c|}
\hline Classe & Táxon & FG & MDL & $\mathrm{BV}$ & $\mathrm{S} / \mathrm{V}$ \\
\hline CHLO & Schroederia indica Philipose & 2cone & 41,3 & 265,7 & 50,1 \\
\hline CHLO & Selenastrum bibraianum Reinsch & mon & 35,0 & $3.237,2$ & 0,7 \\
\hline CHLO & Sphaerocystis planctonica (Koršikov) Bourrelly & esf & 48,0 & 561,2 & 1,3 \\
\hline CHLO & Sphaerocystis schroeteri Chodat & esf & 30,0 & 381,7 & 1,3 \\
\hline CHLO & $\begin{array}{l}\text { Stauridium tetras (Ehrenberg) E. Hegewald (= Pediastrum } \\
\text { tetras (Ehrenberg) Ralfs) }\end{array}$ & cil & 17,8 & 496,6 & 1,1 \\
\hline CHLO & Tetraedron caudatum (Corda) Hansgirg & Pret & 6,8 & 157,2 & 1,2 \\
\hline CHLO & Tetraedron incus (Teiling) G.M. Smith & pir & 4,6 & 33,1 & 3,5 \\
\hline CHLO & Tetraedron minimum (A. Braun) Hansgirg & Pret & 9,1 & 372,6 & 0,9 \\
\hline CHLO & Tetraedron triangulare Koršikov & Ptri & 11,0 & 181,5 & 1,2 \\
\hline CHLO & Tetrallantos lagerheimii Teiling & mon & 40,0 & $1.024,9$ & 0,9 \\
\hline CHLO & Tetrastrum elegans Playfair & Pret & 9,5 & 286,7 & 1,1 \\
\hline CHLO & Tetrastrum glabrum (Y.V. Roll) Ahlstrom \& Tiffany & Pret & 9,0 & 243,0 & 1,1 \\
\hline CHLO & Tetrastrum heteracanthum (Nordstedt) Chodat & Pret & 9,5 & 286,7 & 1,1 \\
\hline CHLO & Tetrastrum Komárekii Hindák & Pret & 8,4 & 202,2 & 1,2 \\
\hline CHLO & Tetrastrum punctatum Ahlstrom \& Tiffany & Pret & 7,3 & 130,4 & 1,4 \\
\hline CHLO & Tetrastrum triangulare (Chodat) Komárek & Pret & 8,3 & 227,1 & 1,1 \\
\hline CHLO & Treubaria crassispina Z.-Y. Hu & esf & 85,0 & 904,8 & 0,7 \\
\hline CHLO & Treubaria schmidlei (Schröder) Fott \& Kovácik & esf & 39,3 & $1.397,1$ & 0,4 \\
\hline CHLO & Treubaria setigera (Archer) G.M. Smith & pir & 13,0 & 102,5 & 2,4 \\
\hline CHLO & Treubaria triapendiculata C. Bernard & pir & 27,3 & 218,7 & 1,9 \\
\hline CHAE & Uronema confervicola Lagerheim & cil & 120,0 & $3.583,8$ & 0,8 \\
\hline CHAE & Uronema elongatum Hodgetts & cil & 91,0 & 643,2 & 1,5 \\
\hline CHLO & Westella botryoides (W. West) De Wildeman & esf & 50,0 & $8.578,7$ & 0,8 \\
\hline CHRY & Bitrichia ollula (Fott) Bourrelly & esf & 33,0 & 477,9 & 0,6 \\
\hline CHRY & Chromulina elegans Doflein & esf & 3,6 & 30,3 & 1,7 \\
\hline CHRY & Chromulina mikroplankton (Pascher) Pascher & esf & 3,0 & 4,2 & 3,0 \\
\hline CHRY & Chromulina minima Doflein & esf & 3,0 & 14,1 & 2,0 \\
\hline CHRY & Chromulina nebulosa Cienkowski & esfo & 10,3 & 263,0 & 0,8 \\
\hline CHRY & Chromulina pseudograndis (Schiller) Matvienko & esf & 30,0 & $8.181,3$ & 0,2 \\
\hline CHRY & Chromulina pygmaea Nygaard & esf & 5,5 & 65,6 & 1,2 \\
\hline CHRY & Chromulina sphaerica Bachmann & esf & 9,0 & 143,8 & 0,9 \\
\hline CHRY & Chrysococcus radians $\mathrm{W}$. Conrad & esf & 14,0 & $1.458,7$ & 0,4 \\
\hline CHRY & Conradiella ovata $\mathrm{H}$. Ettl \& Perman & esf & 11,0 & 268,1 & 0,8 \\
\hline CHRY & Derepyxis amphora A. Stokes & esf & 25,0 & 904,8 & 0,5 \\
\hline CHRY & $\begin{array}{l}\text { Dinobryon divergens var. schauinslandii } \\
\text { (Lemmermann) Brunnthaler }\end{array}$ & esfo & 9,1 & 145,3 & 1,0 \\
\hline CHRY & Lagynion ampullaceum (Stokes) Pascher & esf & 3,0 & 14,1 & 2,0 \\
\hline CHRY & Lagynion macrotrachelum (Stokes) Pascher & esf & 5,5 & 89,3 & 1,1 \\
\hline CHRY & Mallomonas actinoloma E. Takahashi & esf & 9,3 & 223,8 & 0,8 \\
\hline CHRY & Mallomonas apochromatica Conrad & esfo & 45,0 & $7.713,0$ & 0,6 \\
\hline CHRY & Mallomonas miminima Rehfous & esfo & 12,5 & 368,0 & 0,8 \\
\hline
\end{tabular}


Tabela 3 (continuação)

\begin{tabular}{|c|c|c|c|c|c|}
\hline Classe & Táxon & FG & MDL & $\mathrm{BV}$ & $\mathrm{S} / \mathrm{V}$ \\
\hline$\overline{\mathrm{CHRY}}$ & Mallomonas mirabilis Conrad & esfo & 35,0 & $5.935,0$ & 0,6 \\
\hline CHRY & Mallomonas tonsurata Teiling & esfo & 18,0 & 763,4 & 0,6 \\
\hline CHRY & Monas dinobryonis Skuja & cone $+0,5$ esf & 8,0 & 100,5 & 2,9 \\
\hline CHRY & Ochromonas crenata Klebs & esf & 13,8 & 539,5 & 0,6 \\
\hline CHRY & Ochromonas danica E.G. Pringsheim & cone $+0,5$ esf & 9,5 & 390,8 & 3,4 \\
\hline CHRY & Ochromonas margaritata Skuja & esfo & 12,0 & 165,0 & 0,9 \\
\hline CHRY & Ochromonas nana Doflein & esf & 4,0 & 33,5 & 1,5 \\
\hline CHRY & Ochromonas ovalis Doflein & esf & 6,8 & 164,6 & 0,9 \\
\hline CHRY & Ochromonas taeniata Ettl & esf & 5,0 & 65,5 & 1,2 \\
\hline CHRY & Ochromonas variabilis Meyer & esf & 6,4 & 73,6 & 1,2 \\
\hline CHRY & Ochromonas verrucosa Skuja & esf & 20,0 & $4.188,8$ & 0,3 \\
\hline CHRY & Pseudokephyrion hypermaculatum Ettl & esf & 9,0 & 87,1 & 1,1 \\
\hline CHRY & Pseudokephyrion latum (Schiller) Schmid & esf & 7,0 & 179,6 & 0,9 \\
\hline CHRY & Stylochrysalis libera (Fott) Bourrelly & esfo & 18,8 & 455,2 & 0,7 \\
\hline COSC & Aulacoseira ambigua (Grunow) Simonsen var. ambigua & cil & 120,0 & $3.484,2$ & 0,8 \\
\hline COSC & Aulacoseira distans (Ehrenberg) Simonsen & cil & 15,0 & 662,7 & 0,8 \\
\hline COSC & Aulacoseira granulata (Ehrenberg) Simonsen & cil & 103,0 & $2.922,7$ & 0,7 \\
\hline COSC & Aulacoseira granulata var. angustissima (Müller) Simonsen & cil & 16,3 & 480,6 & 1,6 \\
\hline COSC & Cyclotella meneghiniana Kützing & cil & 23,6 & $7.020,5$ & 0,3 \\
\hline COSC & Discostella stelligera (Cleve \& Grunow) Houk \& Klee & cil & 9,5 & 861,4 & 0,6 \\
\hline COSC & Melosira varians C. Agardh & cil & 11,0 & 329,7 & 0,8 \\
\hline CRAS & Codosiga botrytis (Ehrenberg) Saville-Kent & esfo & 2,0 & 2,4 & 3,7 \\
\hline CRAS & Monosiga brevipes Saville-Kent & esf & 4,0 & 35,1 & 1,5 \\
\hline CRAS & Monosiga varians Skuja & esfo & 6,1 & 50,8 & 1,3 \\
\hline CRAS & Salpingoeca ampullacea D. Bicudo \& C. Bicudo & esfo & 9,0 & 117,8 & 1,0 \\
\hline CRAS & Salpingoeca caudiculata D. Bicudo \& C. Bicudo & esfo & 10,0 & 159,7 & 1,0 \\
\hline CRAS & Salpingoeca clarkii Stein & esfo & 7,5 & 53,7 & 1,5 \\
\hline CRAS & Salpingoeca fusiformis Saville-Kent & esfo & 21,7 & 833,7 & 0,6 \\
\hline CRYP & Chilomonas oblonga Pascher & cone $+0,5$ esf & 19,1 & $1.215,1$ & 2,8 \\
\hline CRYP & Chilomonas paramaecium Ehrenberg & cone $+0,5$ esf & 16,5 & 635,0 & 2,7 \\
\hline CRYP & $\begin{array}{l}\text { Cryptomonas brasiliensis A. Castro, C. Bicudo \& } \\
\text { D. Bicudo }\end{array}$ & cone $+0,5$ esf & 12,8 & 550,8 & 3,0 \\
\hline CRYP & Cryptomonas curvata Ehrenberg & cone $+0,5$ esf & 38,8 & $8.225,9$ & 2,7 \\
\hline CRYP & Cryptomonas erosa Ehrenberg & esfo & 20,2 & $1.441,1$ & 0,5 \\
\hline CRYP & Cryptomonas marssonii Skuja & cone $+0,5$ esf & 14,7 & 672,0 & 2,9 \\
\hline CRYP & Cryptomonas nordstedtii (Hansgirg) Senn & esfo & 8,4 & 155,7 & 0,9 \\
\hline CRYP & Cryptomonas obovata Czosnowski & esfo & 15,7 & 452,1 & 0,7 \\
\hline CRYP & Cryptomonas ovata Ehrenberg & esfo & 19,8 & $1.444,2$ & 0,5 \\
\hline CRYP & Cryptomonas phaseolus Skuja & esfo & 11,6 & 272,2 & 0,8 \\
\hline CRYP & Cryptomonas platyuris Skuja & esfo & 39,6 & $5.547,5$ & 0,3 \\
\hline CRYP & Cryptomonas tenuis Pascher & cone $+0,5$ esf & 8,1 & 115,9 & 2,9 \\
\hline CRYP & Cryptomonas tetrapyrenoidosa Skuja & esfo & 23,3 & $2.732,0$ & 0,4 \\
\hline CRYP & Cyathomonas truncata (Fresenius) Fisch & esfo & 12,5 & 320,7 & 0,7 \\
\hline CRYP & Protocryptomonas acuta A. Castro, C. Bicudo \& D. Bicudo & cone $+0,5$ esf & 17,3 & $1.100,6$ & 2,9 \\
\hline CRYP & $\begin{array}{l}\text { Protocryptomonas ellipsoidea Skvortzov ex A. Castro, } \\
\text { C. Bicudo \& D. Bicudo }\end{array}$ & esfo & 10,5 & 269,4 & 0,8 \\
\hline
\end{tabular}


Tabela 3 (continuação)

\begin{tabular}{|c|c|c|c|c|c|}
\hline Classe & Táxon & FG & MDL & BV & $\mathrm{S} / \mathrm{V}$ \\
\hline CRYP & $\begin{array}{l}\text { Protocryptomonas sygmoidea A. Castro, C. Bicudo \& } \\
\text { D. Bicudo }\end{array}$ & cone $+0,5$ esf & 19,6 & $1.138,5$ & 2,8 \\
\hline CRYP & Rhodomonas lacustris Pascher \& Ruttner & cone $+0,5$ esf & 11,0 & 216,0 & 2,8 \\
\hline CRYP & Rhombocystis lacryma Komárek & esfo & 8,0 & 45,8 & 2,2 \\
\hline DINO & Gymnodinium paradoxum A.J. Schilling & esfo & 35,8 & $17.323,3$ & 0,2 \\
\hline DINO & Peridiniopsis cunningtonii Lemmermann & elip & 24,1 & $3.654,8$ & 0,3 \\
\hline DINO & Peridinium cinctum (O.F. Müller) Ehrenberg & esfo & 44,1 & $43.263,2$ & 0,1 \\
\hline DINO & Peridinium gatunense Nygaard & esfo & 40,2 & $33.824,6$ & 0,2 \\
\hline DINO & Peridinium umbonatum F. Stein & esfo & 17,7 & $2.763,6$ & 0,4 \\
\hline DINO & Peridinium willei Huitfeldt-Kaas & esf & 38,0 & $28.731,0$ & 0,2 \\
\hline EUGL & Euglena agilis H.J. Carter & esfo & 30,0 & $1.570,8$ & 0,5 \\
\hline EUGL & Euglena deses Ehrenberg & esfo & 119,1 & $2.969,0$ & 0,7 \\
\hline EUGL & Euglena limnophila Lemmermann & esfo & 65,1 & $2.269,3$ & 0,6 \\
\hline EUGL & Euglena minuta Prescott & esfo & 16,0 & $1.604,4$ & 0,7 \\
\hline EUGL & $\begin{array}{l}\text { Euglena oxyuris Schmarda var. charkoviensis } \\
\text { (Svirenko) P. Bourrelly }\end{array}$ & esfo & 119,0 & $29.611,4$ & 0,2 \\
\hline EUGL & Euglena polymorpha P.A. Dangeard & cone & 45,0 & $1.782,3$ & 0,6 \\
\hline EUGL & Euglena proxima P.A. Dangeard var. piriformis M. Szabados & esfo & 47,0 & $9.843,7$ & 0,3 \\
\hline EUGL & Euglena proxima var. proxima P.A. Dangeard & esfo & 65,5 & $10.772,8$ & 0,3 \\
\hline EUGL & Euglena splendens P.A. Dangeard & esfo & 65,6 & $6.184,6$ & 0,4 \\
\hline EUGL & Euglenaria anabaena (Mainx) Karnkowska \& E.W. Linton & esfo & 21,0 & $1.288,8$ & 0,7 \\
\hline EUGL & Lepocinclis acus (O.F. Müller) Marin \& Melkonian & esfo & 198,7 & $11.297,3$ & 0,6 \\
\hline EUGL & Lepocinclis fusiformis (H.J. Carter) Lemmermann & esfo & 35,4 & $10.792,4$ & 0,2 \\
\hline EUGL & Lepocinclis ovata var. australica (Playfair) Conrad & esfo & 35,0 & $3.577,8$ & 0,3 \\
\hline EUGL & Lepocinclis ovum (Ehrenberg) Lemmermann & esfo & 20,1 & $1.592,2$ & 0,4 \\
\hline EUGL & $\begin{array}{l}\text { Lepocinclis ovum (Ehrenberg) Lemmermann var. } \\
\text { bütschli (Lemmermann) Conrad }\end{array}$ & esfo & 43,6 & $8.985,0$ & 4,8 \\
\hline EUGL & $\begin{array}{l}\text { Lepocinclis ovum (Ehrenberg) Lemmermann var. conica } \\
\text { Allorge \& Lefèvre }\end{array}$ & 2 cone & 43,0 & $8.825,8$ & 9,4 \\
\hline EUGL & $\begin{array}{l}\text { Lepocinclis ovum (Ehrenberg) Lemmermann var. } \\
\text { dimidio-minor (Deflandre) Conrad }\end{array}$ & esfo & 15,0 & 636,2 & 0,6 \\
\hline EUGL & Lepocinclis salina F.E. Fritsch & esfo & 39,0 & $17.791,4$ & 0,2 \\
\hline EUGL & Lepocinclis steinii Lemmermann & esfo & 25,6 & $1.083,6$ & 0,5 \\
\hline EUGL & Menoidium gracile Playfair & esfo & 79,8 & $5.824,2$ & 0,5 \\
\hline EUGL & Phacus acuminatus Stokes & Peli & 19,7 & 607,1 & 1,2 \\
\hline EUGL & Phacus agilis var. okobojiensis Allegre \& Jahn & elip & 18,0 & 678,6 & 0,6 \\
\hline EUGL & Phacus curvicauda Svirenko & Peli & 35,0 & 929,1 & 1,1 \\
\hline EUGL & Phacus ephippion Pochmann & Peli & 119,0 & $1.934,8$ & 2,1 \\
\hline EUGL & Phacus longicauda (Ehrenberg) Dujardin & Peli & 119,5 & $1.852,0$ & 2,1 \\
\hline EUGL & Phacus monilatus Stokes var. suecicus Lemmermann & Peli & 39,7 & 753,2 & 1,7 \\
\hline EUGL & Phacus orbicularis K. Hübner & Peli & 40,0 & $2.827,4$ & 0,8 \\
\hline EUGL & Phacus pleuronectes (O.F. Müller) Nitzsch ex Dujardin & Peli & 42,5 & $2.957,0$ & 0,8 \\
\hline EUGL & Phacus raciborskii Drezepolski & Peli & 27,0 & 135,1 & 2,3 \\
\hline
\end{tabular}


Tabela 3 (continuação)

\begin{tabular}{|c|c|c|c|c|c|}
\hline Classe & Táxon & FG & MDL & $\mathrm{BV}$ & $\mathrm{S} / \mathrm{V}$ \\
\hline$\overline{\text { EUGL }}$ & Phacus tortus (Lemmermann) Skvortzov & Peli & 103,3 & $1.709,4$ & 2,1 \\
\hline EUGL & Strombomonas fluviatilis (Lemmermann) Deflandre & esfo & 30,0 & $1.134,9$ & 0,6 \\
\hline EUGL & Strombomonas verrucosa (E. Daday) Deflandre & esfo & 42,0 & $9.698,1$ & 0,2 \\
\hline EUGL & Trachelomonas abrupta Svirenko & esfo & 22,0 & $1.393,8$ & 0,5 \\
\hline EUGL & Trachelomonas armata (Ehrenberg) F. Stein & esfo & 41,1 & $21.197,1$ & 0,2 \\
\hline EUGL & $\begin{array}{l}\text { Trachelomonas armata (Ehrenberg) F. Stein var. } \\
\text { steinii Lemmermann }\end{array}$ & esfo & 40,0 & $22.808,0$ & 0,2 \\
\hline EUGL & $\begin{array}{l}\text { Trachelomonas armata var. armata (Ehrenberg) F. } \\
\text { Stein f. inevoluta G. Deflandre }\end{array}$ & esfo & 27,0 & $7.478,6$ & 0,2 \\
\hline EUGL & Trachelomonas bacillifera var. globulosa Playfair & esf & 12,3 & 974,3 & 0,5 \\
\hline EUGL & Trachelomonas crebea Kellicott & esfo & 23,0 & $1.592,7$ & 0,4 \\
\hline EUGL & Trachelomonas curta A.M. Cunha & esfo & 16,0 & $1.784,6$ & 0,5 \\
\hline EUGL & $\begin{array}{l}\text { Trachelomonas curta A.M. Cunha var. minima G. } \\
\text { Tell \& Z. Domitrovic }\end{array}$ & esfo & 10,9 & 433,2 & 0,6 \\
\hline EUGL & Trachelomonas granulata Svirenko & esfo & 27,0 & $5.941,2$ & 0,3 \\
\hline EUGL & Trachelomonas hispida (Perty) F. Stein & esfo & 19,3 & $7.054,1$ & 0,2 \\
\hline EUGL & $\begin{array}{l}\text { Trachelomonas hispida (Perty) F. Stein var. } \\
\text { coronata Lemmermann }\end{array}$ & esfo & 22,3 & $3.879,6$ & 0,3 \\
\hline EUGL & $\begin{array}{l}\text { Trachelomonas hispida (Perty) F. Stein var. } \\
\text { crenulatocollis (Maskell) Lemmermann }\end{array}$ & esfo & 32,5 & $6.215,4$ & 0,3 \\
\hline EUGL & Trachelomonas kellogii Skvortzov & esfo & 34,8 & $16.463,3$ & 0,2 \\
\hline EUGL & Trachelomonas lacustris R. Drezepolski & esfo & 26,0 & $2.127,1$ & 0,4 \\
\hline EUGL & Trachelomonas minuscula $\mathrm{R}$. Drezepolski & esf & 7,0 & 179,6 & 0,9 \\
\hline EUGL & Trachelomonas oblonga Lemmermann & esfo & 10,9 & 432,6 & 0,6 \\
\hline EUGL & Trachelomonas planctonica Svirenko var. flexicollis Balech & esfo & 19,8 & $2.546,3$ & 0,4 \\
\hline EUGL & Trachelomonas planctonica var. planctonica Svirenko & esfo & 25,0 & $4.977,5$ & 0,3 \\
\hline EUGL & Trachelomonas robusta Svirenko & esfo & 26,0 & $6.589,0$ & 0,3 \\
\hline EUGL & Trachelomonas sculpta Balech & esf & 21,0 & $4.849,1$ & 0,3 \\
\hline EUGL & Trachelomonas similis A. Stokes & esfo & 24,5 & $3.928,6$ & 0,3 \\
\hline EUGL & Trachelomonas stokesii Drezepolski & esfo & 14,0 & $1.229,6$ & 0,5 \\
\hline EUGL & Trachelomonas verrucosa A. Stokes & esf & 17,5 & $2.806,2$ & 0,3 \\
\hline EUGL & Trachelomonas volvocina (Ehrenberg) Ehrenberg & esf & 12,5 & $1.015,3$ & 0,5 \\
\hline EUGL & $\begin{array}{l}\text { Trachelomonas volvocina (Ehrenberg) Ehrenberg var. } \\
\text { derephora Conrad }\end{array}$ & esf & 12,5 & $1.015,3$ & 0,5 \\
\hline EUGL & Trachelomonas volvocinopsis Svirenko & esf & 15,4 & $1.912,3$ & 0,4 \\
\hline EUST & Pseudocharaciopsis minuta (A. Braun) Hibberd & elip & 10,0 & 51,3 & 1,2 \\
\hline EUST & Vischeria undulata Pascher & esfo & 12,0 & 628,3 & 0,6 \\
\hline FRAG & Synedra rumpens Kützing & Peli & 97,7 & $1.067,9$ & 1,1 \\
\hline FRAG & Ulnaria acus (Kützing) Aboal & Peli & 188,5 & $4.448,2$ & 0,7 \\
\hline FRAG & $\begin{array}{l}\text { Ulnaria delicatissima var. angustissima (Grunow) } \\
\text { Aboal \& Silva }\end{array}$ & Peli & 50,0 & $1.418,7$ & 0,7 \\
\hline FRAG & Ulnaria ulna (Nitzsch) Compère & Peli & 110,0 & $12.684,2$ & 0,7 \\
\hline PRAS & Argillamonas brasiliana Skvortzov & esfo & 11,3 & 206,0 & 0,9 \\
\hline
\end{tabular}


Tabela 3 (continuação)

\begin{tabular}{|c|c|c|c|c|c|}
\hline Classe & Táxon & FG & MDL & $\mathrm{BV}$ & $\mathrm{S} / \mathrm{V}$ \\
\hline PRAS & Fluitomonas centralis Skvortzov ex C. Bicudo & esfo & 6,8 & 5,5 & 3,8 \\
\hline PRAS & Fluitomonas elliptica Skvortzov & elip & 7,5 & 17,7 & 2,0 \\
\hline PRAS & Fluitomonas pyrenoidosa Skvortzov ex C. Bicudo & esfo & 6,8 & 28,7 & 1,8 \\
\hline PRAS & Pedinomonas minutissima Skuja & esfo & 4,0 & 18,8 & 1,9 \\
\hline PRAS & Pedinomonas natantes Skvortzov & esfo & 9,0 & 75,4 & 1,3 \\
\hline PRAS & Protoaceromonas rugosa Skvortzov ex C. Bicudo & esfo & 11,3 & 230,1 & 0,8 \\
\hline PRAS & Protoaceromonas spinosa Skvortzov & esf & 6,0 & 65,5 & 1,2 \\
\hline PRAS & Protochroomonas granulata Skvortzov & esf & 7,0 & 179,6 & 0,9 \\
\hline PRAS & Pseudopedinomonas astigmata (Skvortzov) C. Bicudo & esfo & 10,0 & 214,7 & 0,8 \\
\hline PRAS & Pseudopedinomonas coccinea (Skvortzov) C. Bicudo & esfo & 5,1 & 30,9 & 1,6 \\
\hline PRAS & Pseudopedinomonas hiemalis Skvortzov & esfo & 5,5 & 48,4 & 1,3 \\
\hline PRAS & Pseudopedinomonas oculata (Skvortzov) C. Bicudo & esfo & 7,0 & 33,0 & 1,7 \\
\hline PRYM & Chrysochromulina breviturrita K.H. Nicholls & esf & 10,0 & 523,6 & 0,6 \\
\hline RAPH & Merotricha bacillata Mereschkowsky & esfo & 47,5 & $16.172,4$ & 0,2 \\
\hline RAPH & Vacuolaria tropicalis C. Bicudo \& M.B. Cardoso & esfo & 49,0 & $25.484,1$ & 0,4 \\
\hline RAPH & Vacuolaria virescens Cienkowski & esfo & 74,0 & $22.200,0$ & 0,4 \\
\hline TREB & Actinastrum aciculare Playfair & cone & 16,0 & 24,1 & 5,2 \\
\hline TREB & $\begin{array}{l}\text { Actinastrum aciculare f. minimum (G.E. Huber-Pestalozzi) } \\
\text { P. Compère }\end{array}$ & cone & 12,5 & 18,8 & 5,2 \\
\hline TREB & Actinastrum gracillimum Smith & cil & 14,0 & 253,2 & 3,5 \\
\hline TREB & Actinastrum hantzschii Lagerheim & cone & 11,5 & 78,3 & 2,6 \\
\hline TREB & Botryococcus braunii Kützing & esf & 30,0 & $14.137,2$ & 0,2 \\
\hline TREB & Chlorella vulgaris Beyerinck & esf & 6,5 & 145,8 & 0,9 \\
\hline TREB & Crucigenia tetrapedia (Kirchner) Kuntze & Pret & 6,5 & 89,7 & 1,6 \\
\hline TREB & Crucigenia fenestrata (Schmidle) Schmidle & esfo & 9,6 & 238,6 & 1,3 \\
\hline TREB & Crucigenia neglecta B. Fott \& H. Ettl & esfo & 6,2 & 37,5 & 1,5 \\
\hline TREB & Crucigenia quadrata Morren & Pret & 6,5 & 92,0 & 1,4 \\
\hline TREB & Crucigeniella crucifera (Wolle) Komárek & Pret & 11,0 & 98,3 & 1,8 \\
\hline TREB & Crucigeniella rectangularis (Nägeli) Komárek & Pret & 8,0 & 256,0 & 2,5 \\
\hline TREB & Dicloster acuatus C.-C. Jao, Y.S. Wei \& H.C. Hu & mon & 42,5 & $1.357,8$ & 1,6 \\
\hline TREB & Dictyosphaerium ehrenbergianum Nägeli & esfo & 27,5 & 527,8 & 1,4 \\
\hline TREB & Didymocystis bicellularis (R. Chodat) Komárek & cil & 5,3 & 51,5 & 2,0 \\
\hline TREB & Didymocystis fina Komárek & cil & 5,5 & 45,2 & 2,2 \\
\hline TREB & Didymocystis planctonica Koršikov & elip & 7,1 & 91,3 & 1,1 \\
\hline TREB & Franceia ovalis (Francé) Lemmermann & esfo & 15,0 & 746,6 & 0,5 \\
\hline TREB & Golenkiniopsis longispina (Koršikov) Koršikov & esf & 73,0 & 448,9 & 0,6 \\
\hline TREB & Golenkiniopsis solitaria (Koršikov) Koršikov & esf & 45,0 & 95,4 & 1,1 \\
\hline TREB & $\begin{array}{l}\text { Hegewaldia parvula (Woronichin) T. Proschold, C. Bock, } \\
\text { W. Luo \& L. Krienitz (= Golenkiniopsis parvula) }\end{array}$ & esf & 15,7 & 38,8 & 1,4 \\
\hline TREB & $\begin{array}{l}\text { Keratococcus bicaudatus (A. Braun ex Rabenhorst) } \\
\text { J.B. Petersen }\end{array}$ & 2cone & 16,8 & 87,3 & 22,9 \\
\hline TREB & Keratococcus suecicus Hindák & 2 cone & 40,0 & 94,2 & 80,2 \\
\hline TREB & $\begin{array}{l}\text { Mucidosphaerium pulchellum (H.C. Wood) C. Bock, Proschold } \\
\text { \& Krienitz (= Dictyosphaerium pulchellum H.C. Wood ) }\end{array}$ & esf & 38,3 & 310,9 & 1,4 \\
\hline
\end{tabular}


Tabela 3 (continuação)

\begin{tabular}{|c|c|c|c|c|c|}
\hline Classe & Táxon & FG & MDL & $\mathrm{BV}$ & $\mathrm{S} / \mathrm{V}$ \\
\hline TREB & Nephrochlamys allanthoidea Koršikov & mon & 16,0 & 49,5 & 3,0 \\
\hline TREB & Nephrochlamys subsolitaria (G.S. West) Koršikov & mon & 8,5 & 21,9 & 3,0 \\
\hline TREB & Nephrochlamys willeana (Printz) Koršikov & mon & 10,0 & 31,3 & 2,3 \\
\hline TREB & Nephrocytium agardhianum Nägeli & mon & 19,0 & $2.520,3$ & 0,8 \\
\hline TREB & Nephrocytium schilleri (Kammerer) Comas & esfo & 15,0 & $2.650,7$ & 0,7 \\
\hline TREB & Nephrocytium spirale Beck-Mannagetta & elip & 8,0 & 74,2 & 0,8 \\
\hline TREB & Oocystis borgei J. Snow & esfo & 15,0 & $2.356,2$ & 0,5 \\
\hline TREB & Oocystis lacustris Chodat & esfo & 14,7 & 242,0 & 1,3 \\
\hline ULVO & Ulothrix tenerrima (Kützing) Kützing & cil & 72,5 & $4.211,7$ & 0,6 \\
\hline XANT & Botryochloris minima Pascher & esf & 3,0 & 42,4 & 2,0 \\
\hline XANT & Centritractus belonophorus (W. Schmidle) Lemmermann & cil & 27,9 & $1.152,7$ & 0,6 \\
\hline XANT & Characiopsis aquilonaris Skuja & elip & 7,0 & 11,7 & 1,4 \\
\hline XANT & Characiopsis minima Pascher & elip & 8,3 & 24,7 & 1,7 \\
\hline XANT & $\begin{array}{l}\text { Characiopsis pyriformis (A. Braun) Borzi var. } \\
\text { subsessilis Lemmermann }\end{array}$ & elip & 13,3 & 194,8 & 0,8 \\
\hline XANT & Chloridella cystiformis Pascher in Rabenhorst & esf & 6,0 & 113,1 & 1,0 \\
\hline XANT & Chlorocloster raphidioides Pascher & elip & 15,0 & 58,9 & 1,3 \\
\hline XANT & Dioxys papilioniformis G. Tell \& H. Pizarro & elip & 6,0 & 88,0 & 1,1 \\
\hline XANT & Goniochloris mutica (A. Braun) Fott & Ptri & 8,0 & 66,7 & 1,7 \\
\hline XANT & Heterothrix hormidioides Vischer & cil & 106,0 & 522,8 & 1,9 \\
\hline XANT & Heterothrix stichococcoides Pascher & cil & 160,0 & 604,4 & 2,2 \\
\hline XANT & Isthmochloron lobulatum (Nägeli) Skuja & Pret +8 cone & 40,0 & $4.999,3$ & 0,5 \\
\hline XANT & Monallantus angustus Ettl & cil & 12,7 & 173,1 & 1,1 \\
\hline XANT & Monallantus brevicylindrus Pascher & cil & 8,0 & 128,8 & 1,2 \\
\hline XANT & Nephrodiella minor Pascher & esfo & 5,0 & 23,6 & 1,8 \\
\hline XANT & Nephrodiella semilunaris Pascher & mon & 7,9 & 26,9 & 2,3 \\
\hline XANT & Ophiocytium capitatum Wolle & cil & 10,0 & 282,7 & 0,9 \\
\hline XANT & Ophiocytium maximum Borzì & cil & 18,0 & 286,3 & 1,0 \\
\hline XANT & Ophiocytium parvulum (Perty) A. Braun & cil & 9,0 & 113,1 & 1,2 \\
\hline XANT & Rhizochloris stigmatica Pascher & pir & 13,0 & 624,0 & 1,3 \\
\hline XANT & Tetraedriella jovetti (Bourrelly) Bourrelly & Pret & 8,3 & 285,9 & 1,0 \\
\hline XANT & Tetraplektron bourrellyi Ettl & pir & 20,0 & $2.666,7$ & 0,8 \\
\hline XANT & Tetraplektron laevis (Bourrelly) Ettl & pir & 26,9 & $6.510,1$ & 0,6 \\
\hline XANT & Tetraplektron tribulus (Pascher) Fott & pir & 22,0 & $3.549,3$ & 0,7 \\
\hline XANT & Tribonema minus (Wille) Hazen & cil & 35,0 & 336,7 & 1,4 \\
\hline ZYGN & $\begin{array}{l}\text { Actinotaenium curtum var. curtum f. minus (Rabenhorst) } \\
\text { Teiling ex Croasdale }\end{array}$ & cil & 25,0 & $3.682,7$ & 0,4 \\
\hline ZYGN & Closterium angustatum Kützing ex Ralfs var. angustatum & cil + 2cone & 260,0 & $79.587,2$ & 0,1 \\
\hline ZYGN & $\begin{array}{l}\text { Closterium baillyanum (Brébisson ex Ralfs) Brébisson } \\
\text { var. baillyanum }\end{array}$ & 2 cone tr & 248,0 & $7.135,4$ & 6,8 \\
\hline ZYGN & $\begin{array}{l}\text { Closterium closterioides (Ralfs) Louis \& Peeters } \\
\quad \text { var. closterioides }\end{array}$ & 2 cone tr & 193,7 & $15.380,8$ & 6,0 \\
\hline
\end{tabular}


Tabela 3 (continuação)

\begin{tabular}{|c|c|c|c|c|c|}
\hline Classe & Táxon & FG & MDL & $\mathrm{BV}$ & $\mathrm{S} / \mathrm{V}$ \\
\hline$\overline{\mathrm{ZYGN}}$ & Closterium dianae Ehrenberg ex Ralfs var. dianae f. dianae & Mon & 75,0 & $4.838,5$ & 0,3 \\
\hline ZYGN & Closterium ehrenbergii Meneghini ex Ralfs & cil +2 cone & 225,0 & $216.808,1$ & 0,1 \\
\hline ZYGN & Closterium gracile Brébisson ex Ralfs & cil +2 cone & 131,9 & $2.025,2$ & 1,4 \\
\hline ZYGN & Closterium kuetzingii Brébisson & cil + 2cone & 200,0 & $8.042,5$ & 0,6 \\
\hline ZYGN & Closterium setaceum Ehrenberg ex Ralfs & 2 cil +2 cone & 492,7 & $16.121,0$ & 0,5 \\
\hline ZYGN & Closterium turgidum var. giganteum (Nordstedt) De Toni & cil + 2cone & 900,0 & $6.154 .950,7$ & 0,0 \\
\hline ZYGN & Cosmarium abbreviatum Raciborski & esfo & 11,4 & 634,2 & 0,6 \\
\hline ZYGN & Cosmarium bioculatum Brébisson ex Ralfs & esfo & 22,4 & $2.568,7$ & 0,4 \\
\hline ZYGN & Cosmarium conspersum Ralfs & esfo & 80,0 & $96.510,0$ & 0,1 \\
\hline ZYGN & Cosmarium contractum $\mathrm{O}$. Kirchner & esfo & 26,6 & $4.363,4$ & 0,3 \\
\hline ZYGN & Cosmarium impressulum Elfving & esfo & 33,0 & $9.140,5$ & 0,2 \\
\hline ZYGN & Cosmarium majae Strøm & esfo & 7,8 & 222,9 & 0,9 \\
\hline ZYGN & $\begin{array}{l}\text { Cosmarium margaritatum (P.M. Lundell) Roy \& Bissett var. } \\
\text { margaritatum f. minor (Boldt) W. West \& G.S. West }\end{array}$ & esfo & 39,5 & $29.459,0$ & 0,2 \\
\hline ZYGN & Cosmarium minutum Delponte & esfo & 12,8 & 961,3 & 0,5 \\
\hline ZYGN & Cosmarium pseudoconnatum Nordstedt & esfo & 48,0 & $21.136,7$ & 0,2 \\
\hline ZYGN & Cosmarium regnellii Wille & esfo & 12,0 & 454,0 & 0,6 \\
\hline ZYGN & Cosmarium sphagnicola W. West \& G.S. West & Pret & 10,0 & 500,0 & 0,8 \\
\hline ZYGN & Cosmarium subcucumis f. compressum & esfo & 60,0 & $21.237,2$ & 0,2 \\
\hline ZYGN & Cosmarium subcucumis Schmidle var. subcucumis & esfo & 39,0 & $5.227,6$ & 0,3 \\
\hline ZYGN & Cosmarium sublobulatum (Ralfs) W. Archer & esfo & 9,2 & 339,9 & 0,7 \\
\hline ZYGN & Cosmarium sublobulatum var. brasiliense Borge & esfo & 10,5 & 421,5 & 0,7 \\
\hline ZYGN & $\begin{array}{l}\text { Cosmarium subtumidum Nordstedt var. subtumidum f. minor } \\
\text { Borge }\end{array}$ & elip & 12,0 & 904,8 & 0,3 \\
\hline ZYGN & Cosmarium trilobulatum Reinsch & esfo & 22,0 & $2.359,9$ & 0,4 \\
\hline ZYGN & Cosmocladium pusillum L. Hilse & esfo & 13,5 & 522,8 & 0,6 \\
\hline ZYGN & Desmidium graciliceps (Nordstedt) Lagerheim & cil & 23,0 & $113.659,9$ & 0,3 \\
\hline ZYGN & Desmidium laticeps var. quadrangulare Nordstedt & Pret & 28,0 & $52.136,0$ & 0,4 \\
\hline ZYGN & Euastrum binale var. hians (W. West) W. Krieger & Pret & 15,6 & $1.002,0$ & 0,7 \\
\hline ZYGN & Gonatozygon pilosum Wolle & cil & 105,0 & $5.277,9$ & 0,5 \\
\hline ZYGN & Groenbladia undulata (Nordstedt) K. Förster & cil & 37,5 & $1.446,0$ & 0,7 \\
\hline ZYGN & Haplotaenium minutum (Ralfs) Bando & cil & 138,0 & $13.395,8$ & 0,4 \\
\hline ZYGN & Netrium digitus (Brébisson ex Ralfs) Itzigsohn \& Rothe & esfo & 219,0 & $223.250,0$ & 0,1 \\
\hline ZYGN & Netrium oblongum var. cylindricum W. West \& G.S. West & cil & 21,4 & 387,2 & 0,9 \\
\hline ZYGN & $\begin{array}{l}\text { Staurastrum alternans (Brébisson) Ralfs var. } \\
\text { basichondrum Schmidle f. basichondrum }\end{array}$ & 2 cone tr & 22,0 & $1.943,9$ & 8,7 \\
\hline ZYGN & Staurastrum anatinum var. parvum (W. West) Prescott & 2 cone tr & 26,7 & 744,7 & 0,7 \\
\hline ZYGN & Staurastrum ellipticum var. minus Skuja & 2 cone tr & 21,0 & $2.463,0$ & 0,4 \\
\hline ZYGN & $\begin{array}{l}\text { Staurastrum iversenii var. americanum A.M. Scott } \\
\text { \& R. Grönblad }\end{array}$ & 2 cone tr & 41,0 & 965,5 & 0,5 \\
\hline ZYGN & Staurastrum margaritaceum Meneghini ex Ralfs & $\begin{array}{l}2 \text { cone tr }+ \\
2 \text { cil }\end{array}$ & 22,0 & $2.444,7$ & 0,4 \\
\hline ZYGN & Staurastrum muticum Brébisson ex Ralfs & 2 cone tr & 21,5 & $2.390,8$ & 0,4 \\
\hline
\end{tabular}


Tabela 3 (continuação)

\begin{tabular}{|c|c|c|c|c|c|}
\hline Classe & Táxon & FG & MDL & BV & $\mathrm{S} / \mathrm{V}$ \\
\hline$\overline{\mathrm{ZYGN}}$ & Staurastrum paradoxum Meyen ex Ralfs & 2 cone $\operatorname{tr}$ & 22,0 & $2.488,1$ & 0,3 \\
\hline ZYGN & Staurastrum quadrangulare Brébisson & 2Pret & 18,4 & $3.047,0$ & 0,4 \\
\hline ZYGN & Staurastrum rotula Nordstedt & $\begin{array}{l}2 \text { cone } \operatorname{tr}+ \\
2 \text { cil }\end{array}$ & 64,0 & $6.738,7$ & 0,3 \\
\hline ZYGN & Staurastrum setigerum Cleve var. minus Schmidle & 2 cone tr & 19,5 & 822,3 & 7,2 \\
\hline ZYGN & Staurastrum tetracerum f. trigona P.M. Lundell & $\begin{array}{l}2 \text { cone tr }+ \\
6 \text { cil }\end{array}$ & 10,1 & 694,7 & 0,9 \\
\hline ZYGN & Staurastrum tetracerum Ralfs ex Ralfs & $\begin{array}{c}2 \text { cone tr }+ \\
4 \text { cil }\end{array}$ & 19,4 & 581,5 & 1,1 \\
\hline ZYGN & Staurastrum volans $\mathrm{W}$. West & 2 cone tr & 27,7 & $1.179,1$ & 0,5 \\
\hline ZYGN & Staurodesmus cuspidatus (Brébisson) Teiling & 2 cone tr & 38,0 & $3.445,7$ & 0,3 \\
\hline ZYGN & Staurodesmus dejectus var. dejectus Teiling & 2 cone $\operatorname{tr}$ & 18,1 & $1.559,8$ & 0,4 \\
\hline ZYGN & Staurodesmus extensus var. joshuae (Gutwinski) Teiling & 2 cone tr & 43,0 & 399,4 & 15,1 \\
\hline ZYGN & Staurodesmus incus (Hassal ex Ralfs) Teiling & 2 cone tr & 13,0 & 109,4 & 6,1 \\
\hline ZYGN & Staurodesmus mamillatus (Nordstedt) Teiling & 2 cone tr & 17,0 & 447,8 & 5,2 \\
\hline ZYGN & Staurodesmus triangularis var. minor G.S. West & 2 cone $\operatorname{tr}$ & 3,0 & 24,7 & 5,4 \\
\hline ZYGN & Teilingia granulata (J. Roy \& Bisset) Bourrelly & cil & 150,0 & $11.781,0$ & 0,6 \\
\hline
\end{tabular}

tais como: alta resolução taxonômica, método relativamente barato e simples de aplicar e restrito a poucas fontes de erros que, muitas vezes, estão sob controle do pesquisador. Contudo, a grande desvantagem é a inconsistência do cálculo do biovolume, o que pode ser contornado utilizando os modelos geométricos sugeridos pelos autores. Posteriormente, Sun \& Liu (2003) melhoraram a aplicabilidade dos modelos geométricos de Hillebrand e colaboradores reduzindo o número de parâmetros de linhas microscopicamente medidas, aperfeiçoando as formas e atualizando os modelos. Atualmente, a maioria dos estudos (ex. Soares et al. 2007, Crossetti \& Bicudo 2008, Kruk et al. 2009, Becker et al. 2010, Wyatt et al. 2010, Fonseca \& Bicudo 2011, Borduqui \& Ferragut 2012) que aplicaram o cálculo do biovolume para algas planctônicas e perifíticas utilizaram, basicamente, Hillebrand et al. (1999) e/ou Sun \& Liu (2003), tornando a aplicação do biovolume mais consistente e padronizada.

Vale a pena dizer, porém, que diferenças aparentemente pequenas nas medidas de uma mesma espécie apresentadas por pesquisadores distintos podem gerar grandes discrepâncias no valor final do biovolume, ainda que sejam utilizadas as mesmas formas geométricas. Tais variações são causadas, por exemplo, por diferenças de alguns poucos micrômetros para mais ou para menos resultantes do efeito de halos de luz ou da resolução do equipamento óptico utilizado. Nesse sentido, acredita-se que o uso de listas contendo biovolume das espécies de algas de ecossistemas aquáticos em nível regional possa facilitar os estudos comparativos.

A princípio, recomenda-se que o cálculo do biovolume seja feito, preferencialmente, a partir das medidas dos espécimes locais, para evitar erros decorrentes de diferenças nas dimensões de indivíduos de populações distintas ou mesmo de variações sazonais no tamanho dos representantes das espécies. De fato, alguns táxons são particularmente sensíveis a isto. Fonseca \& Bicudo (2008) registraram, por exemplo, variação sazonal de até duas ordens de magnitude no biovolume de cianobactérias coloniais dos gêneros Microcystis ou Sphaerocavum em represa hipertrófica (Lago das Garças). Desta forma, casos específicos como este devem ser tratados com a maior atenção, a fim de evitar os problemas mencionados anteriormente.

A lista de biovolumes apresentada auxiliará estudos sobre ecologia de algas, pois permitirá a aplicação dessa informação nos trabalhos em andamento na área de estudo, além de servir de modelo comparativo para outros ecossistemas de região tropical. Acredita-se, por fim, que os dados de biovolume apresentados possam ser utilizados em outros locais, desde que as espécies registradas tenham dimensões próximas às citadas no presente trabalho, o que pode ser verificado pelos valores de MDL. Representam um passo para a padronização do cálculo do biovolume no Brasil, permitindo, assim, avançar nos estudos comparativos de ecossistemas. 


\section{Agradecimentos}

Ao projeto "Tipologia, monitoramento e recuperação de corpos d'água da Reserva Biológica do Parque Estadual das Fontes do Ipiranga, São Paulo", financiado pela Fundação de Amparo à Pesquisa do Estado de São Paulo (FAPESP, processos 1999/10695-7 e 2002/00817-2), no qual as teses citadas aqui estiveram inseridas; à Dra Vera L.M. Huszar (MNRJ), pela planilha que serviu de base para cálculo dos biovolumes.

\section{Literatura citada}

Becker, V., Caputo, L., Ordonez, J., Marce, R., Armengol, J., Crossetti, L.O. \& Huszar, V.L.M. 2010. Driving factors of the phytoplankton functional groups in a deep mediterranean reservoir. Water Research 44: 3345-3354.

Bellinger, E.G. \& Sigee, D.C. 2010. Freshwater Algae: Identification and Use as Bioindicators. John Wiley \& Sons, Chichester.

Borduqui, M. \& Ferragut, C. 2012. Factors determining periphytic algae succession in a tropical hypereutrophic reservoir. Hydrobiologia 683: 109-122.

Brasil. 2011. Ministério da Saúde. Portaria no. 2.914 de 12 de dezembro de 2011. Dispõe sobre os procedimentos de controle e de vigilância da qualidade da água para consumo humano e seu padrão de potabilidade. Diário Oficial da União no. 239, Seção 1, Brasília, 14 de dezembro de 2011, pp. 39-46.

Crossetti, L.O. 2006. Estrutura e dinâmica da comunidade fitoplanctônica no período de oito anos em ambiente eutrófico raso (Lago das Garças), Parque Estadual das Fontes do Ipiranga, São Paulo. Tese de Doutorado, Universidade de São Paulo, Ribeirão Preto.

Crossetti, L.O. \& Bicudo, C.E.M. 2008. Adaptations in phytoplankton life strategies to imposed change in a shallow urban tropical eutrophic reservoir, Garças Reservoir, over 8 years. Hydrobiologia 614: 91-105.

Edler, L. 1979. Recomendations for marine biological studies in the Baltic Sea: Phytoplankton and chlorophyll. [s.1.], 33p. (Baltic Marine Biologists).

Ferragut, C. 2004. Respostas das algas perifíticas e planctônicas à manipulação de nutrientes $(\mathrm{N}$ e $\mathrm{P})$ em reservatório urbano (Lago do IAG, São Paulo). Tese de Doutorado, Universidade Estadual Paulista, Rio Claro.

Ferrari, F. 2010. Estrutura e dinâmica da comunidade de algas planctônicas e perifíticas (com Ênfase nas Diatomáceas) em reservatórios oligotrófico e hipertrófico (Parque Estadual das Fontes do Ipiranga, São Paulo). Tese de Doutorado, Universidade Estadual Paulista, Rio Claro.
Figueredo, C.C. \& Giani, A. 2001. Seasonal variation in the diversity and species richness of phytoplankton in a tropical eutrophic reservoir. Hydrobiologia 445: 165-174.

Fonseca, B.M. 2005. Diversidade fitoplanctônica como discriminador ambiental em dois reservatórios rasos com diferentes estados tróficos no Parque Estadual das Fontes do Ipiranga, São Paulo, SP. Tese de Doutorado, Universidade de São Paulo, São Paulo.

Fonseca, B.M. \& Bicudo, C.E.M. 2008. Phytoplankton seasonal variation in a shallow stratified eutrophic reservoir (Garças Pond, Brazil). Hydrobiologia 600: 267-282.

Fonseca, B.M. \& Bicudo, C.E.M. 2010. How important can the presence/absence of macrophytes be in determining phytoplankton strategies in two tropical shallow reservoirs with different trophic status? Journal of Plankton Research 32: 31-46.

Fonseca, B.M. \& Bicudo, C.E.M. 2011. Phytoplankton seasonal and vertical variations in a tropical shallow reservoir with abundant macrophytes (Ninféias Pond, Brazil). Hydrobiologia 665: 229-245.

Giani, A., Bird, D.F., Prairie, Y.T. \& Lawrence, J.F. 2005. Empirical study of cyanobacterial toxicity along a trophic gradient of lakes. Canadian Journal of Fisheries and Aquatic Sciences 62: 2100-2109.

Gosselain, V. \& Hamilton, P. 2000. Algamica: revisions to a key-based computerized counting program for free-living, attached, and benthic algae. Hydrobiologia 438: $139-142$.

Hawkins, P.R., Holliday, J., Kathuria, A. \& Bowling, L. 2005. Change in cyanobacterial biovolume due to preservation by Lugol's Iodine. Harmful Algae 4: 1033-1043.

Hepperle, D., Schmidt-Halewicz, S.E., 2000. Opticount (C). A software tool for the enumeration and biomass determination of plankton organisms and other particles. Win32-Version. http://science.do-mix.de/software opticount.php (acesso em 10.05.2012).

Hillebrand, H., Dürselen, C.-D., Kirschtel, D., Pollingher, U. \& Zohary, T. 1999. Biovolume calculation for pelagic and benthic microalgae. Journal of Phycology 35: 403-424.

Jardim, F.A., Calierei, S.O., Gallinari, P.C. \& Vianna, L.N.L. 2002. Metodologia para a contagem de cianobactérias em células $/ \mathrm{mL}$ - um novo desafio para o analista de laboratório. Engenharia Sanitária e Ambiental 7: 1-3.

Konoplya, B.I. \& Soares, F.S. 2011. New geometric models for calculation of microalgal biovolume. Brazilian Archives of Biology and Technology 54: 527-534. 
Kruk, C., Huszar, V.L.M., Peeters, E.H.M., Bonilla, S., Costa, L., Lurling, M., Reynolds, C.S.\& Scheffer, M. 2009. A morphological classification capturing functional variation in phytoplankton. Freshwater Biology 55: 614-629.

Lopes, M.R., Bicudo, C.E.M. \& Ferragut, C. 2005. Short term spatial and temporal variation of phytoplankton in a shallow tropical oligotrophic reservoir, southeast Brazil. Hydrobiologia 542: 235-247.

Reynolds, C.S. 1984. The Ecology of Freshwater Phytoplankton. Cambridge University Press, Cambridge.

Rott, E. 1981. Some results from phytoplankton counting intercalibrations. Schweizerische Zeitschrift für Hydrologie 43: 34-62.

Sant'Anna, C.L., Azevedo, M.T.P., Werner, V.R., Dogo, C.R., Rios, F.R. \& Carvalho, L.H. 2008. Review of toxic species of Cyanobacteria in Brazil. Algological Studies 126: 251-265.

Soares, M.C.S., Huszar, V.L.M. \& Roland, F. 2007. Phytoplankton dynamics in two tropical rivers with different degrees of human impact (Southeast Brazil). River Research and Applications 23: 698-714.

Stevenson, R.J. 1996. An introduction to algal ecology in freshwater benthic habits. In: R.J. Stevenson, M.L. Bothwell \& R.L. Lowe (eds.). Algal Ecology: freshwater benthic ecosystems. Academic Press, San Diego, pp. 3-30.
Stevenson, R.J. \& Smol, J.P. 2003. Use of Algae in Environmental Assessments. In: J.D. Wehr, \& R.G. Sheath (eds.). Freshwater Algae of North America. Academic Press, California, pp. 775-804.

Sun, J. \& Liu, D. 2003. Geometric models for calculating cell biovolume and surface area for phytoplankton. Journal of Plankton Research 25: 1331-1346.

Taylor, W.D. \& Wetzel, R.G. 1988. Phytoplankton community dynamics in Lawrence Lake of southwestern Michigan. Archiv fur Hydrobiologie 81: 491-532.

Torgan, L.C., Becker, V. \& Rodrigues, S.C. 1998. Volume celular de espécies fitoplanctônicas da Laguna dos Patos, Rio Grande do Sul, Brasil. Nota científica. Biociências 6: 183-186.

Tucci, A. 2002. Sucessão da comunidade fitoplanctônica de um reservatório urbano e eutrófico, São Paulo, SP, Brasil. Tese de Doutorado, Universidade Estadual Paulista, Rio Claro.

Wyatt, K.H., Stevenson, R.J. \& Turetsky, M.R. 2010. The importance of nutrient co-limitation in regulating algal community composition, productivity and algalderived DOC in an oligotrophic marsh in interior Alaska. Freshwater Biology 55: 1845-1860. 